\title{
COVERING CYCLES IN SPARSE GRAPHS
}

\author{
FRANK MOUSSET, NEMANJA ŠKORIĆ, AND MILOŠ TRUJIĆ
}

\begin{abstract}
Let $k \geqslant 2$ be an integer. Kouider and Lonc proved that the vertex set of every graph $G$ with $n \geqslant n_{0}(k)$ vertices and minimum degree at least $n / k$ can be covered by $k-1$ cycles. Our main result states that for every $\alpha>0$ and $p=p(n) \in(0,1]$, the same conclusion holds for graphs $G$ with minimum degree $(1 / k+\alpha) n p$ that are sparse in the sense that

$$
e_{G}(X, Y) \leqslant p|X||Y|+o\left(n p \sqrt{|X||Y|} / \log ^{3} n\right) \quad \forall X, Y \subseteq V(G) .
$$

In particular, this allows us to determine the local resilience of random and pseudorandom graphs with respect to having a vertex cover by a fixed number of cycles. The proof uses a version of the absorbing method in sparse expander graphs.
\end{abstract}

\section{INTRODUCTION}

A classical result of Dirac states that every graph with $n \geqslant 3$ vertices and minimum degree at least $n / 2$ contains a Hamilton cycle, that is, a cycle passing through all vertices of the graph [10]. There exist a vast number of extensions of this theorem, most of which state that every graph satisfying a certain minimum degree condition must have some 'global' structure.

For example, Hajnal and Szemerédi [18] proved that, for every $k \geqslant 2$, the vertex set of every graph with $n$ vertices and minimum degree at least $(k-1) n / k$ can be covered by vertex-disjoint copies of $K_{k}$, provided that $k$ divides $n$. Pósa [12] and Seymour [35] conjectured that, more generally, every graph with $n$ vertices and minimum degree at least $(k-1) n / k$ contains the $(k-1)$ st power of a Hamilton cycle, that is, a Hamilton cycle in which every pair of vertices at distance at most $k-1$ is connected by an edge (the case $k=2$ is Dirac's theorem). This conjecture was first proved for large $n$ by Komlós, Sárközy, and Szemerédi [20], using the regularity method, and later for smaller values of $n$ by Levitt, Sárközy, and Szemerédi [26] and Chau, DeBiasio, and Kierstead [8]. A minimum degree condition ensuring the presence of more general subgraphs, formulated in terms of the chromatic number, is given by the bandwidth theorem of Böttcher, Schacht, and Taraz [7].

The above results all concern graphs with rather large minimum degrees. In the case where the minimum degree can be smaller than $\lfloor n / 2\rfloor$, the graph might not be connected, so one can no longer guarantee any spanning connected subgraph; similarly, the graph might be bipartite, and one cannot guarantee any non-bipartite subgraph. However, such graphs may still have some interesting global properties. The following extension of Dirac's theorem to graphs with minimum degree below $n / 2$ was first conjectured by Enomoto, Kaneko, and Tuza [11] and proved by Kouider and Lonc [21].

Theorem 1.1 ([21]). Let $k \geqslant 2$ be an integer and let $G$ be a graph with $n$ vertices and minimum degree at least $n / k$. Then the vertex set of $G$ can be covered by $k-1$ cycles, edges, or vertices.

We note that if $n=n(k)$ is large enough, then 'cycles, edges, or vertices' can be replaced by 'cycles' (this follows for example from the main result in [4]).

There is a trend in modern combinatorics towards proving sparse analogues of extremal results (see, e.g., Conlon's survey [9]). Our main result in this paper is a sparse analogue of Theorem 1.1.

Date: 18th November 2021.

Research supported by Israel Science Foundation grants 1028/16 and 1147/14, and ERC Starting Grant 633509 (FM), and by grant no. 200021169242 of the Swiss National Science Foundation (MT). 
We use the following natural notion of uniformly sparse graphs, which can be seen as a one-sided version of Thomason's jumbled graphs [39, 40].

Definition $1.2((p, \beta)$-sparse). A graph $G$ is $(p, \beta)$-sparse if for all subsets $X, Y \subseteq V(G)$,

$$
e_{G}(X, Y) \leqslant p|X||Y|+\beta \sqrt{|X||Y|} .
$$

It is well known that for every $p=p(n) \leqslant 0.99$, the Erdős-Rényi random graph $G_{n, p}$ is $(p, O(\sqrt{n p}))$-sparse w.h.p. ${ }^{1}[23]$. With this definition, our main result reads as follows.

Theorem 1.3. For every integer $k \geqslant 2$ and every $\alpha>0$, there exists a positive $\eta(\alpha, k)$ such that the following holds for all sufficiently large $n$, all $p \in(0,1]$, and all $\beta \leqslant \eta n p / \log ^{3} n$. Let $G$ be $a(p, \beta)$-sparse graph with $n$ vertices and minimum degree at least $(1 / k+\alpha) n p$. Then the vertex set of $G$ can be covered by $k-1$ cycles.

The minimum degree $(1 / k+\alpha) n p$ cannot be much improved. Indeed, assume $\log ^{6} n / n \ll p \ll 1$ and let $G$ be a graph consisting of $k$ vertex-disjoint copies of $G_{n / k, p}$. Then w.h.p. $G$ has minimum degree at least $(1 / k-o(1)) n p$ and is $(p, \beta)$-sparse with $\beta=O(\sqrt{n p})=o\left(n p / \log ^{3} n\right)$, but cannot be covered by $k-1$ cycles. A very similar construction shows that the upper bound on $\beta$ in our result is optimal up to the logarithmic factors. To see this, take any $\log n / n \ll p \ll 1$ and consider the random graph $G$ given by the union of $k$ vertex-disjoint copies of $G_{n / k, q}$, where $q=(1+2 k \alpha) p$. Then $G$ cannot be covered by $k-1$ cycles but, at the same time, w.h.p. it has minimum degree at least $(1 / k-o(1)) n q \geqslant(1 / k+\alpha) n p$ and is $(q, O(\sqrt{n q}))$-sparse. The latter means that for all $X, Y \subseteq V(G)$,

$$
e(X, Y) \leqslant q|X||Y|+O(\sqrt{n q|X||Y|}) \leqslant p|X||Y|+O(n p \sqrt{|X||Y|}),
$$

using $p|X||Y| \leqslant n p \sqrt{|X||Y|}$, so $G$ is in fact $(p, O(n p))$-sparse.

Our main motivation for studying the problem from Theorem 1.3 is the connection to the local resilience of sparse random and pseudorandom graphs. The systematic study of this notion was initiated by Sudakov and $\mathrm{Vu}[38]$ and, since then, the topic has garnered considerable attention (see, e.g., [1, 2, 3, 25, 29, 36] and the surveys [6, 37]).

Definition 1.4 (Local resilience). Let $\mathcal{P}$ be a monotone ${ }^{2}$ graph property and let $G$ be a graph in $\mathcal{P}$. The local resilience of $G$ with respect to $\mathcal{P}$ is defined as the maximum $r \in[0,1]$ such that, for every $H \subseteq G$ satisfying $\operatorname{deg}_{H}(v)<r \operatorname{deg}_{G}(v)$ for all $v \in V(G)$, we have $G-H \in \mathcal{P}$.

For example, Theorem 1.1 implies that the local resilience of $K_{n}$ with respect to having a vertex-cover by $k-1$ cycles is at least $(k-1) / k-o(1)$, which is easily seen to be optimal (consider a disjoint union of $k$ cliques of size $n / k)$. Since $G_{n, p}$ is w.h.p. $(p, O(\sqrt{n p})$ )-sparse and has degrees concentrated around $n p$, Theorem 1.3 has the following consequence for the local resilience of random graphs.

Theorem 1.5. Let $k \geqslant 2$ be an integer and let $p=p(n)$ be such that $p \gg \log ^{6} n / n$. Then the local resilience of $G_{n, p}$ with respect to having a vertex-cover by $k-1$ cycles is w.h.p. $(k-1) / k \pm o(1)$.

Indeed, it is not difficult to see that $(k-1) / k+o(1)$ is an upper bound (consider a random partition of the vertex set into $k$ parts). Note that if $p \ll \log n / n$, then w.h.p. $G_{n, p}$ has an unbounded number of connected components and thus no vertex-cover by $k-1$ cycles. We believe that with a bit more care, one could improve the lower bound on $p$ in the above theorem to $p \gg \log ^{4} n / n$, using essentially the same proof strategy. However, there is a natural barrier in our method that prevents us from going down all the way to $\log n / n$ (which is the threshold for

\footnotetext{
${ }^{1}$ With high probability, that is, with probability tending to 1 as $n \rightarrow \infty$.

${ }^{2} \mathrm{~A}$ graph property $\mathcal{P}$ is monotone if it is preserved under adding edges.
} 
having a vertex-cover by a constant number of cycles). Doing so would require new ideas and techniques.

Note also that the case $k=2$ in Theorem 1.5 corresponds to the problem of determining the local resilience $G_{n, p}$ with respect to Hamiltonicity. This question was resolved independently by Montgomery [27] and by Nenadov, Steger, and the third author [32], who showed that the local resilience of $G_{n, p}$ with respect to Hamiltonicity is w.h.p. $1 / 2 \pm o(1)$ whenever $p=$ $(\log n+\log \log n+\omega(1)) / n$.

Our last result concerns pseudorandom graphs. An $(n, d, \lambda)$-graph is a $d$-regular graph with $n$ vertices for which all eigenvalues of the adjacency matrix, with the exception of the largest one, are bounded in absolute value by $\lambda$. It follows from the expander mixing lemma that every $(n, d, \lambda)$-graph is $(d / n, \lambda)$-sparse. Thus, Theorem 1.3 has the following consequence.

Theorem 1.6. For every integer $k \geqslant 2$ and every $\alpha>0$, there exists a positive $\eta(\alpha, k)$ such that the following holds for all sufficiently large $n$. Let $G$ be an $(n, d, \lambda)$-graph with $\lambda \leqslant \eta d / \log ^{3} n$. Then the local resilience of $G$ with respect to having a vertex-cover by $k-1$ cycles is $(k-1) / k \pm \alpha$.

Again, it is not difficult to see that $(k-1) / k+o(1)$ is an upper bound. Theorem 1.6 generalises a result of Sudakov and $\mathrm{Vu}$ [38] stating that every $(n, d, \lambda)$-graph $G$ with $\lambda \leqslant d / \log ^{2} n$ has local resilience at least $1 / 2-o(1)$ with respect to Hamiltonicity.

Theorems 1.5 and 1.6 are, to the best of our knowledge, the first positive results on the local resilience of (pseudo)random graphs where the local resilience is significantly larger than 1/2. We believe the methods introduced in this paper could be used to tackle different problems allowing such high resilience.

1.1. Methods and techniques. The proof of Theorem 1.3 combines several techniques for embedding large structures into (pseudo)random graphs and their subgraphs. The focal point is the absorbing method, first introduced under this name by Rödl, Rucinski, and Szemerédi [33], but already used implicitly in earlier works of Erdős, Gyárfás, and Pyber [13] and Krivelevich [22].

We now give a simplified account of how this method approaches the problem of embedding a spanning graph $S$ (in our case, a spanning union of $k-1$ cycles) into a graph $G$. First, one reserves a small subset $R$ of the vertex set of $G$, called the reservoir; frequently, this is simply a uniformly random subset of small size. Then one embeds a certain highly structured graph $A$, called the absorber, into $G[V(G) \backslash R]$, such that the following holds: suppose that we embed a fixed subgraph $S^{\prime} \subseteq S$ into $G[V(G) \backslash V(A)]$ in such a way that all vertices outside of $R \cup V(A)$ are covered; then there exists a completion of the embedding of $S^{\prime}$ to an embedding of $S$. We remark that, usually, it is very difficult to control which vertices of $R$ are used by the embedding of $S^{\prime}$, so this property relies on a careful choice of $A$ (we think of $A$ as 'absorbing' the vertices in $R$ not covered by the embedding of $S^{\prime}$ ). In this way, the problem of embedding the spanning graph $S$ is reduced to the (often easier) problem of embedding the non-spanning graph $S^{\prime}$. A method similar to the one described in this paragraph has been successfully applied to numerous problems in (random) graph theory (see, e.g., [14, 15, 17, 24, 26, 28, 30]).

The first step towards carrying out the above approach is to figure out the structure of the absorber. In our case, one of the main issues to overcome is that the graph $G$ might be bipartite, which means that, in order to have any hope of embedding the absorber into $G[V(G) \backslash R]$, the absorber must be bipartite as well. This creates several technical challenges. Most importantly, we cannot use the common approach of building an absorber by stringing together many singlevertex absorbers, as absorbing only a single vertex may upset the delicate balance between the two parts of the bipartition; rather, we need to be able to absorb two vertices at a time (one from each part of the bipartition). This is done using a variation of a trick of Montgomery [28]. A consequence of this approach is that our absorber is only able to absorb subsets of $R$ that contain the same number of vertices from each part of the (hypothetical) bipartition. 
Even after deciding upon a suitable structure for the absorber, we still face the issue of actually embedding this structure into $G[V(G) \backslash R]$. In the context of sparse pseudorandom graphs, this is usually done by exploiting the expansion properties of the graph to show that one can connect prescribed pairs of vertices or edges by disjoint copies of a given fixed graph $F$ (for example, a path of length $\log n$ ). This statement is usually referred to as the Connecting Lemma, though its precise formulation depends on the nature of $S$. The actual absorber is then embedded by multiple uses of this lemma. A difficulty that arises in our setting (and that is not a problem when the minimum degree is at least $(1 / 2+o(1)) n p)$ is that the graph $G$ that we are dealing with might not be a very good expander at all - in fact, if $k$ is large, then $G$ might have a large number of connected components, implying that there are fairly small sets (of size roughly $n / k$ ) that do not expand at all. An important step in our proof is to show that $G$ can nevertheless be partitioned into at most $k-1$ subgraphs, each having strong enough expansion properties to embed an absorber, all without sacrificing much of the minimum degree. We refer to this as the Partitioning Lemma.

In order to complete the proof, we cover each of these expanding subgraphs by systems of disjoint paths, leaving uncovered only vertices in the reservoir and the absorber. This is done using a standard application of the sparse regularity lemma in conjunction with the recent bootstrapping argument of Nenadov and the second author [31]. Finally, the absorber is used to connect each of these systems of disjoint paths into a cycle (and picking up the uncovered vertices in the reservoir along the way).

We believe that some of these techniques are likely to be of use for other, related problems concerning the structure of uniformly sparse graphs satisfying a minimum degree condition. In particular, the Partitioning Lemma and Connecting Lemma that we prove are quite general statements that are largely unrelated to the concrete problem that is solved in this paper.

1.2. Organisation of the paper. The paper is structured as follows. In Section 2 we introduce the notion of expander graphs and state several useful properties of such graphs. Furthermore, we mention some of the more standard tools we use, namely Haxell's criterion for matchings in hypergraphs and Szemerédi's regularity lemma for sparse graphs and related concepts. In Section 3 we reduce Theorem 1.3 to a version in which one can assume that the graph is an expander graph. We also state all the necessary 'big gun' lemmas used in order to prove it and subsequently give a proof of Theorem 3.2 modulo those lemmas. Each of the following Sections $4-8$ are fully dedicated to the proof of one of the lemmas.

1.3. Notation. We use standard graph theoretic notation. In particular, given a graph $G, V(G)$ and $E(G)$ denote the sets of vertices and edges of $G$, respectively. We write $v(G)=|V(G)|$ and $e(G)=|E(G)|$. For a subset $X \subseteq V(G)$, we denote by $G[X]$ the subgraph induced by the vertex set $X$. For two (not necessarily disjoint) subsets $X, Y \subseteq V(G)$, we write $e_{G}(X, Y)$ for the number of pairs in $X \times Y$ that form an edge, and $e_{G}(X)$ for the number of edges in $X$. Note that $e_{G}(X, X)=2 e_{G}(X)$. Furthermore, we denote by $N_{G}(X, Y)$ the set of all neighbours in $Y$

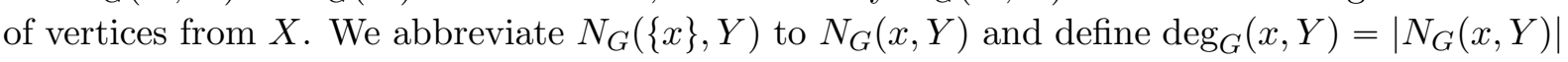
and $\operatorname{deg}_{G}(x)=\operatorname{deg}_{G}(x, V(G))$. We say that a path $P$ connects two vertices $x, y$ if $x$ and $y$ are its endpoints, and say that $P$ is an $x y$-path. The length of a path is defined as the number of edges in it. For $\ell \in \mathbb{N}$, we denote by $N_{G}^{\ell}(X, Y)$ the set of vertices $y \in Y$ for which there exists a path $P$ of length at most $\ell$ connecting $y$ to some $x \in X$ and whose internal vertices are in $Y$; in particular $N_{G}^{1}(X, Y)=N_{G}(X, Y)$. Again, we abbreviate $N_{G}^{\ell}(\{x\}, Y)=N_{G}^{\ell}(x, Y)$. If $X$ and $Y$ are disjoint, then $G[X, Y]$ is the induced bipartite subgraph with parts $X$ and $Y$, and the density of the pair $(X, Y)$ is $d_{G}(X, Y)=e_{G}(X, Y) /(|X||Y|)$. In all of the above notations, we may omit the subscript $G$ when it is clear which graph we are talking about. 
For an integer $n$ we write $[n]=\{1, \ldots, n\}$ and for $a, b, \varepsilon \in \mathbb{R}$, we write $a \in(1 \pm \varepsilon) b$ to denote $(1-\varepsilon) b \leqslant a \leqslant(1+\varepsilon) b$. We use the standard asymptotic notation $o, O, \omega$, and $\Omega$. The logarithm function is always used with the natural base $e$. We suppress floors and ceilings whenever they are not crucial. Finally, we use the convention that if the statement of (say) Lemma 3.6 features a value named $C$, then we may elsewhere write $C_{3.6}$ to denote this value.

\section{EXPANSION AND OTHER PRELIMINARIES}

A simple but important property of $(p, \beta)$-sparse graphs is that every set $A$ of vertices with degree drastically above $|A| p$ must expand by a significant amount. This is the content of our first lemma.

Lemma 2.1. Let $p \in[0,1]$, let $\alpha, \beta>0$, and let $G$ be a $(p, \beta)$-sparse graph on $n$ vertices. Assume $A \subseteq V(G)$ is a subset such that $\operatorname{deg}(a) \geqslant|A| p+\alpha$ np for all $a \in A$. Then

$$
e(A, V(G) \backslash A) \geqslant(\alpha n p-\beta)|A| .
$$

In particular, if $A$ is not empty, then there exists a vertex $a \in A$ such that $\operatorname{deg}(a, V(G) \backslash A) \geqslant$ $\alpha n p-\beta$.

Proof. Set $B=V(G) \backslash A$. Recalling the definition of $e(\cdot, \cdot)$ our assumption implies

$$
e(A, B)=e(A, V(G))-e(A, A) \geqslant|A|^{2} p+|A| \cdot \alpha n p-e(A, A) .
$$

On the other hand, as $G$ is $(p, \beta)$-sparse,

$$
e(A, A) \leqslant|A|^{2} p+\beta|A| .
$$

Combining these inequalities gives $e(A, B) \geqslant(\alpha n p-\beta)|A|$, and the last assertion follows simply by averaging.

In particular, if $G$ is a $(p, o(n p))$-sparse graph with minimum degree $\Omega(n p)$, then the above lemma shows that all small enough linear-sized subsets of vertices expand by a factor $\Omega(n p)$. An important role in the proof is played by graphs in which also the larger sets of vertices have this property. We make the following definition.

Definition 2.2 ( $q$-expander). Let $q>0$. A graph $G$ is a $q$-expander if for every partition $V(G)=V_{1} \cup V_{2}$, we have

$$
e\left(V_{1}, V_{2}\right) \geqslant q\left|V_{1}\right|\left|V_{2}\right| \text {. }
$$

Informally, we think of a $(p, o(n p))$-sparse graph $G$ as being a 'good expander' if it is a $q$ expander for some $q=\Omega(p)$. One can see that this is essentially best possible, since the definition of a $(p, o(n p))$-sparse graph implies that $e\left(V_{1}, V_{2}\right) \leqslant p\left|V_{1}\right|\left|V_{2}\right|+\beta \sqrt{\left|V_{1}\right|\left|V_{2}\right|}=(1+o(1)) p\left|V_{1}\right|\left|V_{2}\right|$ for every partition $V=V_{1} \cup V_{2}$ into sets of linear size.

The following simple lemma allows us to assume without loss of generality that our expanders are bipartite, which turns out to be convenient later on in the proof.

Lemma 2.3. Let $q>0$. Every $q$-expander $G$ contains a spanning bipartite $(q / 2)$-expander as a subgraph.

Proof. Let $V(G)=A \cup B$ be a partition of the vertex set of $G$ that maximises $e_{G}(A, B)$. We claim that $H=G[A, B]$ is a $(q / 2)$-expander. Since we assume that $G$ is a $q$-expander, it is enough to show that for any partition of the vertices into non-empty sets $X$ and $Y$, we have $e_{H}(X, Y) \geqslant e_{G}(X, Y) / 2$. 
To see this, define the sets $V_{1}=X \cap A, V_{2}=X \cap B, V_{3}=Y \cap A$, and $V_{4}=Y \cap B$, and note that

$$
\begin{aligned}
2 e_{H}(X, Y)+e_{G}\left(V_{1} \cup V_{4}, V_{2} \cup V_{3}\right) & =e_{G}\left(V_{1} \cup V_{2}, V_{3} \cup V_{4}\right)+e_{G}\left(V_{1} \cup V_{3}, V_{2} \cup V_{4}\right) \\
& =e_{G}(X, Y)+e_{G}(A, B) ;
\end{aligned}
$$

here, the first equality can be verified by writing $e_{H}(X, Y)=e_{G}\left(V_{1}, V_{4}\right)+e_{G}\left(V_{2}, V_{3}\right)$ and observing that for all $1 \leqslant i \leqslant j \leqslant 4$, both sides of the equality count each edge of $G\left[V_{i}, V_{j}\right]$ the same number of times. The maximal choice of $(A, B)$ ensures that $e_{G}\left(V_{1} \cup V_{4}, V_{2} \cup V_{3}\right) \leqslant e_{G}(A, B)$. This implies the desired inequality $e_{H}(X, Y) \geqslant e_{G}(X, Y) / 2$.

2.1. Matchings in hypergraphs. The following theorem due to Haxell has recently seen a surge of applications in problems concerning embedding (spanning) structures into sparse graphs. It is similar to Hall's theorem in spirit, providing a condition for the existence of a perfect matching in certain hypergraphs.

Theorem 2.4 (Haxell's criterion [19]). Let $A$ and $B$ be disjoint sets and let $\mathcal{H}=(A \cup B, E)$ be an $r$-uniform hypergraph such that $|A \cap e|=1$ and $|B \cap e|=r-1$ for every edge $e \in E$. Suppose that for every choice of subsets $S \subseteq A$ and $Z \subseteq B$ such that $|Z| \leqslant(2 r-3)(|S|-1)$, there is an edge $e \in E$ intersecting $S$ but not $Z$. Then $\mathcal{H}$ contains an $A$-saturating matching (that is, a collection of disjoint hyperedges whose union contains A).

2.2. Sparse regularity lemma. Given a graph $G$ and $\varepsilon, p>0$, we say that a pair $(X, Y)$ of disjoint subsets $X, Y \subseteq V(G)$ is $(\varepsilon, p)$-regular if for all subsets $X^{\prime} \subseteq X$ and $Y^{\prime} \subseteq Y$ with $\left|X^{\prime}\right| \geqslant \varepsilon|X|$ and $\left|Y^{\prime}\right| \geqslant \varepsilon|Y|$, we have $\left|d\left(X^{\prime}, Y^{\prime}\right)-d(X, Y)\right| \leqslant \varepsilon p$. We say that the pair $(X, Y)$ is $(\varepsilon, p)$-lower-regular if for all $X^{\prime}$ and $Y^{\prime}$ as above, we have $d\left(X^{\prime}, Y^{\prime}\right) \geqslant(1-\varepsilon) p$.

A partition $V(G)=V_{0} \cup \cdots \cup V_{t}$ is called an $(\varepsilon, p)$-regular partition with exceptional class $V_{0}$ if $\left|V_{0}\right| \leqslant \varepsilon n,\left|V_{1}\right|=\cdots=\left|V_{t}\right| \leqslant n / t$, and all but at most $\varepsilon t^{2}$ pairs $\left(V_{i}, V_{j}\right)$ with $1 \leqslant i<j \leqslant t$ are $(\varepsilon, p)$-regular.

Lemma 2.5 (Sparse regularity lemma [34]). For all $\varepsilon, m>0$, there exists $M(\varepsilon, m)$ such that for every graph $G$ on at least $M$ vertices, there exists an $(\varepsilon, p)$-regular partition $\left(V_{i}\right)_{i=0}^{t}$ of $V(G)$, where $p=e(G) /\left(\begin{array}{l}n \\ 2\end{array}\right)$ is the density of $G$ and $m \leqslant t \leqslant M$.

Lastly, we need a lemma due to Gerke, Kohayakawa, Rödl, and Steger [16] stating that in an $(\varepsilon, p)$-lower-regular pair, almost all subsets of size at least $D / p$, for some $D>0$, inherit lower-regularity, with slightly weaker parameters.

Lemma 2.6 (Corollary 3.8 in [16]). For all $\varepsilon^{\prime}, \delta \in(0,1)$, there exist positive constants $\varepsilon_{0}\left(\varepsilon^{\prime}, \delta\right)$ and $D\left(\varepsilon^{\prime}\right)$ such that the following holds for all $0<\varepsilon \leqslant \varepsilon_{0}$ and $p \in(0,1)$. Suppose $\left(V_{1}, V_{2}\right)$ is an $(\varepsilon, p)$-lower-regular pair and $q_{1}, q_{2} \geqslant D p^{-1}$. Then the number of pairs $\left(Q_{1}, Q_{2}\right)$ with $Q_{i} \subseteq V_{i}$ and $\left|Q_{i}\right|=q_{i}(i=1,2)$ that are $\left(\varepsilon^{\prime}, p\right)$-lower-regular is at least

$$
\left(1-\delta^{\min \left\{q_{1}, q_{2}\right\}}\right)\left(\begin{array}{c}
\left|V_{1}\right| \\
q_{1}
\end{array}\right)\left(\begin{array}{c}
\left|V_{2}\right| \\
q_{2}
\end{array}\right) .
$$

\section{Proof of Theorem 1.3}

The proof of Theorem 1.3 involves several different ingredients. In this section, we gather the necessary definitions and key lemmas, whose proofs we postpone to the subsequent sections. We then show how these lemmas can be used to deduce our main result.

Recall, our goal is to show that if $G$ is a $(p, \beta)$-sparse graph with $n$ vertices, minimum degree at least $(1 / k+\alpha) n p$, and $\beta \leqslant \eta n p / \log ^{3} n$, then the vertex set of $G$ can be covered by $k-1$ cycles. It turns out that if $G$ is an expander, then it has all kinds of good connectivity properties that 
make it easier to cover it by cycles. However, it is not hard to see that if $k>2$, then $G$ need not even be connected; in particular, it need not be a good expander.

The first step of the proof deals with this problem. For a graph $G$ and $\xi \geqslant 0$, let us write $\delta_{\xi}(G)$ for the maximal integer $d$ such that all but at most $\xi v(G)$ vertices $v$ of $G$ satisfy $\operatorname{deg}_{G}(v) \geqslant d$. Note that $\delta_{0}(G)$ is simply the minimum degree $\delta(G)$ of $G$. On the other hand, $\delta_{\xi}(G)$ for a small constant $\xi>0$ is a sort of 'essential minimum degree' of $G$ possessed by a $(1-\xi)$-fraction of the vertices.

Lemma 3.1 (Partitioning Lemma). For all $c, \alpha, \xi \in(0,1)$, there exist positive $\gamma(\alpha, \xi, c)$ and $\eta(\alpha, \xi, c)$ such that the following holds for all sufficiently large $n$. Let $p \in(0,1)$ and $\beta \leqslant \eta n p$, and let $G$ be a $(p, \beta)$-sparse graph on $n$ vertices with minimum degree at least $(c+\alpha) n p$. Then there exists a partition $V(G)=V_{1} \cup \cdots \cup V_{\ell}$ into $1 \leqslant \ell<1 / c$ parts such that, for every $i \in[\ell]$,

(i) $G\left[V_{i}\right]$ is a $\gamma p$-expander,

(ii) $\delta\left(G\left[V_{i}\right]\right) \geqslant c^{2} n p$,

(iii) $\delta_{\xi}\left(G\left[V_{i}\right]\right) \geqslant(c+\alpha-\xi) n p$.

The proof of this lemma is given in Section 7. Informally, it says that if $G$ has minimum degree slightly above $c n p$, then it can be partitioned into fewer than $1 / c$ good expanders, each of which essentially still has the same minimum degree as $G$ (and each of which has minimum degree at least $\left.c^{2} n p\right)$. This means that from now on, instead of working with an arbitrary $(p, \beta)$-sparse graph with minimum degree $\delta(G) \geqslant(1 / k+\alpha) n p$, we can work with a $(p, \beta)$-sparse expander graph satisfying $\delta_{\xi}(G) \geqslant(1 / k+\alpha-\xi) n p$, where $\xi$ is an arbitrarily small positive constant. Luckily for us, the fact that we go from a graph with $\delta(G) \geqslant(c+\alpha) n p$ to a graph with $\delta_{\xi}(G) \geqslant(c+\alpha-\xi) n p$ does not pose any insurmountable difficulty. Theorem 1.3 now follows easily from the following 'robust' version specialised to expander graphs.

Theorem 3.2. For every integer $k \geqslant 2$ and all $\alpha, \gamma \in(0,1)$, there exists a positive $\eta(\alpha, \gamma, k)$, such that the following holds for all sufficiently large $n$. Let $p \in(0,1]$ and $\beta \leqslant \eta n p / \log ^{3} n$. Then every $(p, \beta)$-sparse $\gamma p$-expander $G$ on $n$ vertices satisfying

$$
\delta(G) \geqslant 2 \alpha n p \quad \text { and } \quad \delta_{\alpha / 128}(G) \geqslant(1 / k+\alpha) n p
$$

has a vertex cover by $k-1$ cycles.

Proof of Theorem 1.3. Without loss of generality we may assume that $\alpha>0$ is small enough, in particular smaller than $1 /\left(2 k^{2}\right)$. Let $\xi=\alpha / 129, \alpha^{\prime}=\alpha-\xi, \gamma=\gamma_{3.1}(\alpha, \xi, 1 / k), \eta^{\prime}=$ $\min _{2 \leqslant i \leqslant k}\left\{\eta_{3.2}\left(\alpha^{\prime}, \gamma, i\right)\right\}$, and $\eta=\min \left\{\alpha \eta^{\prime} / 2, \eta_{3.1}(\alpha, \xi, 1 / k)\right\}$. Let $G=(V, E)$ be a $(p, \beta)$-sparse graph with $n$ vertices, minimum degree $(1 / k+\alpha) n p$, and $\beta \leqslant \eta n p / \log ^{3} n$. We apply Lemma 3.1 with $1 / k$ (as $c$ ) to obtain some $1 \leqslant \ell<k$ and a partition $V=V_{1} \cup \cdots \cup V_{\ell}$ such that, for every $i \in[\ell]$,

(i) $G\left[V_{i}\right]$ is a $\gamma p$-expander,

(ii) $\delta\left(G\left[V_{i}\right]\right) \geqslant n p / k^{2} \geqslant 2 \alpha n p$,

(iii) $\delta_{\xi}\left(G\left[V_{i}\right]\right) \geqslant(1 / k+\alpha-\xi) n p$.

Let $n_{i}:=\left|V_{i}\right|$ and $k_{i}:=\left\lceil k \cdot n_{i} / n\right\rceil$, for every $i \in[\ell]$. Then, by our choice of constants,

$$
\delta_{\alpha^{\prime} / 128}\left(G\left[V_{i}\right]\right)=\delta_{\xi}\left(G\left[V_{i}\right]\right) \geqslant\left(1 / k+\alpha^{\prime}\right) n p \geqslant\left(1 / k_{i}+\alpha^{\prime}\right) n_{i} p .
$$

Next, $(i i)$ and the fact that $G$ is $(p, \beta)$-sparse imply that

$$
\alpha\left|V_{i}\right| n p \leqslant e\left(V_{i}, V_{i}\right) \leqslant\left|V_{i}\right|^{2} p+\beta\left|V_{i}\right|=\left|V_{i}\right|\left(\left|V_{i}\right| p+\beta\right),
$$

from which we deduce, using $\beta \leqslant \eta n p / \log ^{3} n$ for large enough $n$, that $n_{i} \geqslant(\alpha / 2) n$. In particular, we have $\beta \leqslant \eta n p / \log ^{3} n \leqslant \eta^{\prime} n_{i} p / \log ^{3} n_{i}$, by our choice of $\eta$. Since $G\left[V_{i}\right]$ is a subgraph of $G$, it is clearly also $(p, \beta)$-sparse. 
It now follows from Theorem 3.2 applied with $\alpha^{\prime}$ (as $\alpha$ ) that each graph $G\left[V_{i}\right]$ can be covered by $k_{i}-1$ cycles. Since

$$
\sum_{i=1}^{\ell}\left(k_{i}-1\right)=\sum_{i=1}^{\ell}\left\lceil k \cdot n_{i} / n\right\rceil-1<\sum_{i=1}^{\ell} k \cdot n_{i} / n=k,
$$

this shows that also $G$ can be covered by $k-1$ cycles, as required.

It thus remains to prove Theorem 3.2. As one might expect, the proof makes heavy use of the fact that the graph $G$ is a $\gamma p$-expander. The main ingredient is the Connecting Lemma which allows us to connect many given pairs of vertices in $G$ using short disjoint paths. In order to make this precise, we start with the following definition.

Definition 3.3 ((M,W, $\ell$-matching). Let $G$ be a graph and let $W \subseteq V(G)$ be a subset of the vertices. Let $M$ be a multigraph with $V(M) \subseteq V(G) \backslash W$. Then an $(M, W, \ell)$-matching in $G$ is a collection $\left\{P_{e}: e \in E(M)\right\}$ of internally vertex-disjoint paths in $G$ where for every edge $e=\{u, v\} \in E(M)$, the path $P_{e}$ is a $u v$-path of length at most $\ell$ whose internal vertices all lie in the set $W$.

The multigraph $M$ can be thought of as prescribing which pairs of vertices of $G$ ought to be connected by how many paths; an $(M, W, \ell)$-matching is then a system of internally vertexdisjoint paths of length at most $\ell$ connecting the prescribed vertices in the prescribed manner, such that the internal vertices of the paths all lie in the set $W$. The proof of the Connecting Lemma is deferred to Section 5 .

Lemma 3.4 (Connecting Lemma). For every $\gamma \in(0,1)$ and $\Delta>0$, there exists a positive $C(\gamma, \Delta)$ such that the following holds for all sufficiently large $n$. Let $p \in(0,1)$ and $\beta>0$, let $G$ be a $(p, \beta)$-sparse graph on $n$ vertices, and let $U, W \subseteq V(G)$ be disjoint subsets such that:

(i) $G[W]$ is a $\gamma p$-expander,

(ii) $|W| \geqslant C \beta \sqrt{\log n} / p$, and

(iii) every vertex $u \in U$ satisfies $\operatorname{deg}(u, W) \geqslant \gamma|W| p$.

Let $\ell=\lceil 30 \log n /(\gamma \log \log n)\rceil$. Then for every multigraph $M$ with $V(M) \subseteq U$, at most $|W| /(C \ell)$ edges, and maximum degree at most $\Delta$, there exists an $(M, W, \ell)$-matching in $G$.

One important technical property that we make use of is that most not too small subsets of a good expander again induce good expanders, albeit with a slightly weaker parameter.

Lemma 3.5 (Inheritance Lemma). For every $\gamma \in(0,1)$, there exist positive $\gamma_{1}(\gamma)$ and $C(\gamma)$ such that the following holds for all sufficiently large $n$. Let $p \in(0,1)$ and $\beta>0$, let $G$ be $a(p, \beta)$ sparse $\gamma p$-expander on $n$ vertices, and let $r$ be a positive integer such that $r p \geqslant C \cdot \max \{\log n, \beta\}$. Then the number of subsets $R \subseteq V(G)$ of size $r$ for which $G[R]$ is a $\gamma_{1} p$-expander is at least $\left(1-n^{-1}\right)\left(\begin{array}{l}n \\ r\end{array}\right)$.

The proof of the lemma is based on the sparse regularity lemma. We postpone it to Section 4 . Here we just remark that the proof gives a dependence in the order of $\gamma_{1}=\Theta\left(\gamma^{4}\right)$, but we have no reason to believe that this is optimal. An interesting question, unrelated to the topic of this paper, is whether it is possible to achieve a dependence of the form $\gamma_{1}=\Omega(\gamma)$.

The proof of Theorem 3.2 relies on the absorbing method. The main idea of this approach is to embed a small auxiliary structure (an absorber) into the graph $G$ that allows us to reduce the problem of covering $G$ by $k-1$ cycles to a simpler problem. In our case, this simpler problem is to show that there exist subgraphs $P_{1}, \ldots, P_{k-1} \subseteq G$ covering the vertices of $G$, where each $P_{i}$ is a vertex-disjoint union of $O\left(n / \log ^{3} n\right)$ paths. This simpler problem can then be solved using a method based on the sparse regularity lemma. 
Definition 3.6 ((X,Y)-absorber). Let $X$ and $Y$ be disjoint sets of vertices. An $(X, Y)$-absorber is a graph $H$ with two designated vertices $a$ and $b$ (called the endpoints of the absorber) such that $X \cup Y \subseteq V(H) \backslash\{a, b\}$ and such that, for all subsets $X^{\prime} \subseteq X$ and $Y^{\prime} \subseteq Y$ with $\left|X^{\prime}\right|=\left|Y^{\prime}\right|$, $H$ contains an $a b$-path $P$ with $V(P)=V(H) \backslash\left(X^{\prime} \cup Y^{\prime}\right)$ (i.e., an ab-path using all vertices of $H$ with the exception of exactly the vertices in $\left.X^{\prime} \cup Y^{\prime}\right)$.

With this definition we can state the Absorbing Lemma whose proof is presented in Section 6.

Lemma 3.7 (Absorbing Lemma). For every $\gamma \in(0,1)$, there exists a positive $C(\gamma)$ such that the following holds for all sufficiently large $n$. Let $p \in(0,1)$ and $\beta>0$, let $G=(A, B, E)$ be a bipartite $(p, \beta)$-sparse graph on $n$ vertices, and let $U, W \subseteq V(G)$ be disjoint subsets such that:

(i) $G[W]$ is a $\gamma p$-expander,

(ii) $|W| \geqslant C \cdot \max \left\{\log n / p, \beta \sqrt{\log n} / p,|U| \log ^{2} n\right\}$, and

(iii) $|U| \geqslant 2$ and every vertex $u \in U$ satisfies $\operatorname{deg}(u, W) \geqslant \gamma|W| p$.

Then $G[U \cup W]$ contains a $(U \cap A, U \cap B)$-absorber with one endpoint in $W \cap A$ and another in $W \cap B$.

Often when dealing with embedding problems in sparse (pseudorandom) graphs it is not too difficult to embed a desired structure that covers all but $\varepsilon n$ vertices of the graph. With the next lemma we reduce this leftover significantly, and what is more, require that only the majority of vertices have the 'correct' degree for the structure to be embedded. The proof relies on a standard application of the sparse regularity lemma combined with a trick of Nenadov and the second author and is presented in Section 8.

Lemma 3.8 (Embedding Lemma). For every integer $k \geqslant 2$ and every $\alpha>0$, there exists a positive $\eta(\alpha, k)$ such that the following holds for all sufficiently large $n$. Let $p \in(0,1)$ and $\beta \leqslant \eta n p$, and let $G$ be a $(p, \beta)$-sparse graph on $n$ vertices such that

$$
\delta(G) \geqslant 2 \alpha n p \quad \text { and } \quad \delta_{\alpha / 32}(G) \geqslant(1 / k+\alpha) n p .
$$

Then the vertices of $G$ can be covered by $k-1$ path forests $P_{1}, \ldots, P_{k-1}$ that contain at most $\max \{\beta / p, \log n / p\}$ paths each.

With all the 'big guns' at hand we are ready to prove Theorem 3.2.

3.1. Overview. The proof follows a standard strategy relying on the absorbing method: (1) choose a partition $V(G)=V^{\prime} \cup U \cup W$ into sets of appropriate size uniformly at random; (2) use the Absorbing Lemma to find an absorber $H$ in $G[U \cup W]$; (3) use the Embedding Lemma to cover the vertices of $G\left[V^{\prime} \cup W\right]$ not in $H$ by $k-1$ path forests each consisting of at most $n / \log ^{3} n$ paths; (4) for every forest, use the Connecting Lemma over $G[U]$ to combine all its paths into a cycle; and (5) use the absorbing property of $H$ to take in the unused vertices of $G[U]$. One of the main issues we need to deal with while executing this strategy is that for $k \geqslant 3$ the graph $G$ can be bipartite. This introduces several complications.

First of all, in order to find an absorber $H$, the underlying graph $G[U \cup W]$ needs to be bipartite and $G[W]$ a $\gamma p$-expander; even more, for the Connecting Lemma we need $G[U]$ to be an expander as well. In case $k=2$ this is circumvented by choosing a random equipartition of both $U=$ $U_{A} \cup U_{B}$ and $W=W_{A} \cup W_{B}$ taking $F$ to be the bipartite graph between corresponding colour classes. As the (essential) minimum degree of $F[U]$ is in this case around $(1 / 2+o(1))|U| p / 2$, a simple calculation shows that $F[U]$ is a $\gamma_{1} p$-expander, for a suitable choice of $\gamma_{1}$. The same argument applies for $F[W]$. The second issue lies in the fact that, when using the Connecting Lemma across $F[U]$, we need to use exactly the same number of vertices of both $U_{A}$ and $U_{B}$ in order for the 'absorbing property' of $H$ to apply. In case $k=2$ this is not a problem, as all 
vertices $v \in V(G)$ have a significant portion of their degree into both $U_{A}$ and $U_{B}$ in $G$. One can then ensure that the vertices of $U_{A}$ and $U_{B}$ are used in a balanced manner.

More serious problems start to occur when $k \geqslant 3$. For a start, the above strategy of choosing a random equipartition of $U$ and $W$ simply does not work as the obtained bipartite graph is not necessarily an expander. Before partitioning $V(G)$ we first apply Lemma 2.3 to the whole graph $G$, to find a spanning bipartite $(\gamma p / 2)$-expander $F \subseteq G$ on colour classes $A$ and $B$. Next, a random partition is chosen into sets $V^{\prime} \cup U \cup W$ as above, and the Inheritance Lemma (Lemma 3.5) implies $F[U]$ and $F[W]$ are w.h.p. both a $\gamma_{1} p$-expander. However, in this case we cannot guarantee that there is even a single vertex with significant portion of its degree into both $U \cap A$ and $U \cap B$-recall, $G$ may be bipartite itself and $F=G$ potentially! Hence, if in step (4) from above the Connecting Lemma is applied over $F[U \cap A, U \cap B]$ blindly, it may result in an imbalance in the number of vertices used, as we cannot control which of the vertices (the ones given as $V(M)$ to Lemma 3.4) have neighbours in $U \cap A$ and which in $U \cap B$. It may be that all $(k-1) n / \log ^{3} n$ pairs of vertices we are trying to connect have neighbours only in, say, $U \cap A$, resulting in $(k-1) n / \log ^{3} n$ more vertices of $U \cap A$ being used than those of $U \cap B$, since all paths in $F[U]$ with both endpoints in $U \cap A$ are of even length. Luckily, as $k \geqslant 3$, we can reuse some vertices across the $k-1$ cycles we are trying to find, which gives us additional flexibility.

The plan is to find sets $Q_{A}$ and $Q_{B}$, in $V(G) \backslash(U \cup W)$, each of size roughly $k n / \log ^{3} n$, such that all vertices of $Q_{A}$ in $G$ have a large neighbourhood in $U \cap A$ and all vertices of $Q_{B}$ have a large neighbourhood in $U \cap B$. The Connecting Lemma is then used to connect all these vertices into a single path $P_{Q}$, before covering the remainder $V^{\prime} \backslash V\left(P_{Q}\right)$ by $k-1$ path forests, each containing at most $n / \log ^{3} n$ paths. We connect $P_{Q}$ with the first path forest through $F[U]$ to get a cycle $C_{1}$ and then, while connecting the remaining forests into cycles $C_{2}, \ldots, C_{k-1}$, we can reuse the vertices of $P_{Q}$ by adding a carefully chosen subset of them to the pairs in $V(M)$ we aim to connect by the Connecting Lemma. This can be done so that exactly the same number of vertices of $U \cap A$ and $U \cap B$ is used, allowing us to use the 'absorbing property' of $H$ to finally complete the embedding.

Proof of Theorem 3.2. Without loss of generality we may assume $0<p \leqslant 1 / 2$. Given $k, \alpha$, and $\gamma$, let $\gamma_{1}=\min \left\{\alpha / 1024, \gamma / 1024, \gamma_{1} 3.5(\gamma / 2)\right\}, \varepsilon=\min \left\{\alpha / 1024, \gamma_{1} / 64\right\}$,

$$
C=\max \left\{30 / \varepsilon^{2}, C_{3.4}\left(\gamma_{1}, 2\right), C_{3.4}\left(\gamma_{1}^{2} / 8,2\right), C_{3.5}(\gamma / 2), C_{3.7}\left(\gamma_{1} / 2\right)\right\},
$$

and $\eta=\varepsilon / C^{2}$. Suppose that $G=(V, E)$ is a $(p, \beta)$-sparse graph with minimum degree at least $2 \alpha n p$ and $\delta_{\alpha / 128}(G) \geqslant(1 / k+\alpha) n p$. Note that, since $\beta \leqslant \eta n p / \log ^{3} n$ and $\delta(G) \geqslant 2 \alpha n p$, the definition of $(p, \beta)$-sparse graphs applied to a vertex $v$ and its neighbourhood $N_{G}(v)$ implies $p \geqslant \eta^{-1} \log ^{4} n / n$, with room to spare.

The Hamiltonian case, $k=2$. Let $V=V^{\prime} \cup U \cup W$ be a partition of $V$ chosen uniformly at random such that

$$
|U|=\left\lfloor\frac{\varepsilon n}{C \log ^{2} n}\right\rfloor, \quad|W|=\lfloor\varepsilon n\rfloor, \quad \text { and } \quad\left|V^{\prime}\right|=n-|U|-|W| .
$$

Hence, from the bounds on $p$ and $\beta$, we have

$$
|U|,|W|,\left|V^{\prime}\right| \geqslant C \cdot \max \{\beta \sqrt{\log n} / p, \log n / p\},
$$

by our choice of $\eta$. As an easy consequence of Chernoff's inequality and the union bound, with high probability

$$
\operatorname{deg}_{G}(v, Z) \geqslant(2 \alpha-\varepsilon)|Z| p \quad \text { and } \quad \delta_{\alpha / 126}(G[Z]) \geqslant(1 / 2+3 \alpha / 4)|Z| p,
$$

for all $v \in V(G)$ and $Z \in\left\{V^{\prime}, U, W\right\}$. For the remainder of the proof we fix such a good choice of sets $V^{\prime}, U$, and $W$. 
Let $U=U_{A} \cup U_{B}$ and $W=W_{A} \cup W_{B}$ be partitions with $\left|U_{A}\right|=\lfloor|U| / 2\rfloor$ and $\left|W_{A}\right|=\lfloor|W| / 2\rfloor$, and let $F:=G\left[U_{A} \cup W_{A}, U_{B} \cup W_{B}\right]$ a bipartite graph such that the following holds:

(P1) $F[U]$ and $F[W]$ are both a $\gamma_{1} p$-expander,

(P2) $\operatorname{deg}_{F}(v, Z) \geqslant(\alpha / 2)|Z| p$ and $\delta_{\alpha / 120}(F[Z]) \geqslant(1 / 4+\alpha / 2)|Z| p$, for all $v \in U \cup W$ and $Z \in\{U, W\}$

(P3) for every $v \in V(G), \min \left\{\operatorname{deg}_{G}\left(v, U_{A}\right), \operatorname{deg}_{G}\left(u, U_{B}\right)\right\} \geqslant \gamma_{1}|U| p$.

If such partitions are chosen uniformly at random then Chernoff's inequality and the union bound, together with (1) and (3), show that w.h.p. (P2) and (P3) hold. Fix such a choice of sets. We show that these imply (P1) as well.

We only show that $F[U]$ is a $\gamma_{1} p$-expander, as an analogous argument works for $F[W]$. Let $X \subseteq U$ be of size $|X| \leqslant|U| / 2$. If $|X| \leqslant(\alpha / 4)|U|$, then from (P2) by applying Lemma 2.1 with $\alpha / 4$ (as $\alpha), F[U]$ (as $G$ ), and $X$ (as $A$ ) we obtain

$$
e_{F}(X, U \backslash X) \geqslant((\alpha / 4)|U| p-\beta)|X| \geqslant(\alpha / 8)|X||U| p \geqslant \gamma_{1}|X||U| p,
$$

since $\beta=o(|U| p)$. On the other hand, if $(\alpha / 4)|U|<|X| \leqslant|U| / 2$, from (P2) we have

$$
e_{F}(X, U \backslash X) \geqslant\left(|X|-\frac{\alpha}{120}|U|\right)(1 / 4+\alpha / 2)|U| p-2 e_{G}\left(X \cap U_{A}, X \cap U_{B}\right) .
$$

Then, $G$ being $(p, \beta)$-sparse further gives

$$
e_{F}(X, U \backslash X) \geqslant(1 / 4+\alpha / 2)|X||U| p-\frac{\alpha}{120}(1 / 4+\alpha / 2)|U|^{2} p-|X|^{2} p / 2-\beta|X| .
$$

An easy case distinction between $|X|<|U| / 4$ and $|U| / 4 \leqslant|X| \leqslant|U| / 2$ in both cases shows (with room to spare) $e_{F}(X, U \backslash X) \geqslant \gamma_{1}|X||U| p$, as desired.

Given such a graph $F$, for $X \in\{A, B\}$, we say that a vertex $v \in V(G)$ is $X$-expanding if $\operatorname{deg}_{G}\left(v, U_{X}\right) \geqslant \gamma_{1}|U| p$. We say that a pair $\{u, v\}$ of vertices $u, v \in V(G)$ is an $X$-pair if $u$ and $v$ are both $X$-expanding. Note that a vertex can be both $A$-expanding and $B$-expanding.

We apply the Absorbing Lemma (Lemma 3.7) with $\gamma_{1}$ (as $\gamma$ ) and $F$ (as $G$ ) to obtain a $\left(U_{A}, U_{B}\right)$-absorber $H$ in the graph $F[U \cup W]$ with one endpoint $a \in W_{A}$ and the other $b \in W_{B}$. Indeed, (P1), (1) and (2), and (P2) in that order verify Lemma $3.7(i)-(i i i)$.

Let $V^{\prime \prime}:=V^{\prime} \cup(W \backslash V(H))$. Observe that $|W \backslash V(H)| \leqslant \varepsilon n$. Hence, we get for all $v \in V^{\prime \prime}$

$$
\operatorname{deg}_{G}\left(v, V^{\prime \prime}\right) \geqslant \operatorname{deg}_{G}\left(v, V^{\prime}\right) \stackrel{(3)}{\geqslant}(2 \alpha-\varepsilon)\left|V^{\prime}\right| p \geqslant \frac{2 \alpha-\varepsilon}{1+2 \varepsilon}\left|V^{\prime \prime}\right| p \geqslant \alpha\left|V^{\prime \prime}\right| p .
$$

Similarly, all $v \in V^{\prime \prime}$ that previously satisfied $\operatorname{deg}_{G}\left(v, V^{\prime}\right) \geqslant(1 / 2+3 \alpha / 4)\left|V^{\prime}\right| p$, now satisfy

$$
\operatorname{deg}_{G}\left(v, V^{\prime \prime}\right) \geqslant \frac{1 / 2+3 \alpha / 4}{1+2 \varepsilon}\left|V^{\prime \prime}\right| p \geqslant(1 / 2+\alpha / 2)\left|V^{\prime \prime}\right| p,
$$

due to our choice of $\varepsilon$. Lastly, the set of vertices in $V^{\prime \prime}$ violating (5) is of size at most $(\alpha / 126)\left|V^{\prime}\right|+$ $|W| \leqslant(\alpha / 64)\left|V^{\prime \prime}\right|$. Therefore, we can apply the Embedding Lemma (Lemma 3.8) with $\alpha / 2$ (as $\alpha)$ to the graph $G\left[V^{\prime \prime}\right]$ to obtain a path forest $P_{1}$ that contains at most $\max \{\beta / p, \log n / p\} \leqslant$ $n / \log ^{3} n$ paths and covers all vertices of $V^{\prime \prime}$.

Let $m$ denote the total number of paths in $P_{1}$. Take an arbitrary ordering of these $m$ paths and let us denote the endpoints of the $i$-th path by $s_{i}$ and $t_{i}$, for all $i \in[m]$; note that a path may be only a single vertex, in which case $s_{i}=t_{i}$. Since every $v \in V^{\prime \prime}$ has degree at least $\gamma_{1}|U| p$ into both $U_{A}$ and $U_{B}$ (by (P3)), by sequentially choosing for each vertex whether we make it $A$-expanding or $B$-expanding, that is, artificially removing the edges to the other colour class of $F[U]$, we can make the following set of pairs have exactly the same number of $A$-pairs as $B$-pairs:

$$
\mathcal{P}=\left\{\left\{b, s_{1}\right\},\left\{t_{i}, s_{i+1}\right\}_{i \in[m-1]},\left\{t_{m}, a\right\}\right\} .
$$

Finally, we apply the Connecting Lemma (Lemma 3.4) to the graph obtained from $G$ by substituting $G[U]$ by $F[U]$, and with $\gamma_{1}($ as $\gamma), V(G) \backslash U$ (as $\left.U\right), U$ (as $\left.W\right), \ell=30 \log n /\left(\gamma_{1} \log \log n\right)$, 
and the multigraph $M$ with the vertex set

$$
V(M)=\{a, b\} \cup\left\{s_{i}, t_{i}\right\}_{i \in[m]}
$$

and the edge set $E(M)=\mathcal{P}$. Clearly, $\Delta(M) \leqslant 2$ and $e(M) \leqslant m+1 \leqslant 2 n / \log ^{3} n \leqslant|U| /(C \ell)$. Therefore, we obtain an ab-path $P^{\prime}$ covering all vertices of $V^{\prime \prime}$. Crucially, $\left|V\left(P^{\prime}\right) \cap U_{A}\right|=$ $\left|V\left(P^{\prime}\right) \cap U_{B}\right|$, ensured by the argument above.

Let $A^{\prime}$ and $B^{\prime}$ denote the set of vertices in $U_{A}$ and $U_{B}$, respectively, belonging to $V\left(P^{\prime}\right)$. By definition (see Definition 3.6), $H$ contains an ab-path $P$ with $V(P)=V(H) \backslash\left(A^{\prime} \cup B^{\prime}\right)$, and substituting $H$ with $P$ together with $P^{\prime}$ closes a cycle $C_{1}$ which covers all vertices of $G$, as required.

The general case, $k \geqslant 3$. In this case, due to low (essential) minimum degree, splitting $W$ and $U$ uniformly at random into colour classes as above is not enough to guarantee the obtained bipartite graph is an expander. On top of that, the whole graph $G$ may be bipartite and a property like (P3) cannot hold. We use a slightly different approach in this case.

Let $F \subseteq G$ be a spanning bipartite $(\gamma p / 2)$-expander with colour classes $A$ and $B$ obtained by applying Lemma 2.3 to $G$. Let $V=V^{\prime} \cup U \cup W \cup Q \cup Y$ be a partition of $V$ chosen uniformly at random such that

$$
|U|=\left\lfloor\frac{\varepsilon n}{C \log ^{2} n}\right\rfloor, \quad|W|=|Q|=|Y|=\lfloor\varepsilon n\rfloor, \quad \text { and } \quad\left|V^{\prime}\right|=n-|U|-|W|-|Q|-|Y| .
$$

Observe that, from the bounds on $p$ and $\beta$, we have

$$
|U|,|W|,|Q|,|Y|,\left|V^{\prime}\right| \geqslant C \cdot \max \{\beta \sqrt{\log n} / p, \log n / p\},
$$

as before. Since $\delta(F) \geqslant(\gamma / 2) p(n-1)$, as an easy consequence of Chernoff's inequality and the union bound, with high probability

$$
\operatorname{deg}_{F}(v, Z) \geqslant \gamma_{1}|Z| p, \operatorname{deg}_{G}(v, Z) \geqslant(2 \alpha-\varepsilon)|Z| p, \text { and } \delta_{\alpha / 126}\left(G\left[V^{\prime}\right]\right) \geqslant(1 / k+3 \alpha / 4)\left|V^{\prime}\right| p,
$$

for every $v \in V(G)$ and $Z \in\left\{V^{\prime}, U, W, Q, Y\right\}$. Additionally, from the Inheritance Lemma (Lemma 3.5) with probability at least $1-6 n^{-1}$ we have that all of $G[Q], G[Y], G[U \cup Q]$, $F[U], F[W], F[U \cup W]$ are a $\gamma_{1}$ p-expander. For the remainder of the proof we fix such a good partition.

By the Absorbing Lemma (Lemma 3.7) applied with $\gamma_{1}$ (as $\gamma$ ) and $F$ (as $G$ ) there is a $(U \cap A, U \cap B$ )-absorber $H$ in the graph $F[U \cup W]$ with endpoints $a \in W \cap A$ and $b \in W \cap B$. Indeed, $F[W]$ being a $\gamma_{1} p$-expander, (6) and (7), and (8) in that order establish Lemma $3.7(i)-$ (iii).

As before, given $U \cap A$ and $U \cap B$ we say that a pair $\{u, v\}$ of vertices $u, v \in V(G)$ is an $A$-pair if $u$ and $v$ are both $A$-expanding and a $B$-pair if both are $B$-expanding. In order to gain some flexibility on the number of $A$-pairs and $B$-pairs we make use of the following claim.

Claim 3.9. There exist $Q_{A}, Q_{B} \subseteq Q$ of size $\left|Q_{A}\right|,\left|Q_{B}\right|=\left\lfloor k n / \log ^{3} n\right\rfloor$ such that

(a) $\operatorname{deg}_{G}(v, U \cap A) \geqslant\left(\gamma_{1}^{2} / 8\right)|U| p$ for all $v \in Q_{A}$, and

(b) $\operatorname{deg}_{G}(v, U \cap B) \geqslant\left(\gamma_{1}^{2} / 8\right)|U| p$ for all $v \in Q_{B}$.

Proof. We show only the first assertion as the second one follows analogously. Let $U_{A}:=U \cap A$ and $U_{B}:=U \cap B$. We first show that both of these sets have size at least $\gamma_{1}|U| / 4$. Indeed, if we assume that, say, $\left|U_{A}\right|<\gamma_{1}|U| / 4$, we have from the fact that $F[U]$ is a $\gamma_{1} p$-expander, and thus $\delta(F[U]) \geqslant \gamma_{1}(|U|-1) p$,

$$
\gamma_{1}|U|^{2} p / 3 \leqslant e_{F}\left(U_{A}, U_{B}\right) \leqslant e_{G}\left(U_{A}, U_{B}\right) \leqslant \gamma_{1}|U|^{2} p / 4+\beta|U| / 2,
$$

where the upper bound follows from $G$ being $(p, \beta)$-sparse. This leads to a contradiction as $\beta \leqslant \eta n p / \log ^{3} n=o(|U| p)$. 
Let $Q_{A} \subseteq Q$ be defined as

$$
Q_{A}:=\left\{v \in Q: \operatorname{deg}_{G}\left(v, U_{A}\right) \geqslant\left(\gamma_{1}^{2} / 8\right)|U| p\right\},
$$

and suppose towards contradiction $\left|Q_{A}\right| \leqslant n / \log ^{2} n$. Using the fact that $G[U \cup Q]$ is a $\gamma_{1} p$ expander and $\left|U_{A}\right| \geqslant\left(\gamma_{1} / 4\right)|U|$, we get

$$
\left(\gamma_{1}^{2} / 4\right)|U|\left|Q \cup U_{B}\right| p \leqslant e_{G}\left(U_{A}, Q \cup U_{B}\right) \leqslant e_{G}\left(U_{A}, U_{B}\right)+e_{G}\left(U_{A}, Q_{A}\right)+\left(\gamma_{1}^{2} / 8\right)|Q||U| p .
$$

As $\beta \leqslant \eta n p / \log ^{3} n,\left|U_{A}\right|,\left|U_{B}\right|=\Theta\left(n / \log ^{2} n\right)$, and $|Q|=\varepsilon n$, from $G$ being $(p, \beta)$-sparse, we have

$$
e_{G}\left(U_{A}, U_{B}\right) \leqslant(1+o(1))\left|U_{A}\right|\left|U_{B}\right| p \quad \text { and } \quad e_{G}\left(U_{A}, Q_{A}\right) \leqslant o(|U||Q| p) .
$$

Lastly, as $\left|U_{B}\right|=o(|Q|)$, the whole right hand side in (9) can be bounded by $\left(3 \gamma_{1}^{2} / 16\right)|Q||U| p$, which leads to a contradiction. In conclusion, $\left|Q_{A}\right|>n / \log ^{2} n$. This implies there exist sets $Q_{A}, Q_{B}$ of size $\left\lfloor k n / \log ^{3} n\right\rfloor$ as desired.

Take an arbitrary ordering $\left\{v_{1}, \ldots, v_{q}\right\}$ of the vertices in $Q_{A} \cup Q_{B}$, where $q:=\left|Q_{A} \cup Q_{B}\right|$. We apply the Connecting Lemma (Lemma 3.4) to $G$ with $\gamma_{1}\left(\right.$ as $\gamma$ ), $Q_{A} \cup Q_{B}$ (as $U$ ), $Y$ (as $W$ ), and the multigraph $M$ defined as

$$
V(M)=Q_{A} \cup Q_{B} \quad \text { and } \quad E(M)=\left\{\left\{v_{i}, v_{i+1}\right\}_{i \in[q-1]}\right\} .
$$

Since $G[Y]$ is a $\gamma_{1}$ p-expander, by (7) and (8) all the assumptions Lemma $3.4(i)-(i i i)$ are satisfied. Perhaps the least obvious is the bound on $e(M)$ which holds as $q \leqslant 2 k n / \log ^{3} n \leqslant|Y| /(C \log n)$ (see (7)). We obtain a $v_{1} v_{q}$-path $P_{Q}$ which contains all vertices of $Q_{A} \cup Q_{B}$.

Let $W^{\prime}:=W \backslash V(H), Y^{\prime}:=Y \backslash V\left(P_{Q}\right), Q^{\prime}:=Q \backslash\left(Q_{A} \cup Q_{B}\right)$, and $V^{\prime \prime}:=V^{\prime} \cup W^{\prime} \cup Q^{\prime} \cup Y^{\prime}$. One easily checks, similarly as in (4) and (5), that $\delta\left(G\left[V^{\prime \prime}\right]\right) \geqslant \alpha\left|V^{\prime \prime}\right| p$ and $\delta_{\alpha / 64}\left(G\left[V^{\prime \prime}\right]\right) \geqslant$ $(1 / k+\alpha / 2)\left|V^{\prime \prime}\right| p$. Consequently, we apply the Embedding Lemma (Lemma 3.8) with $\alpha / 2$ (as $\alpha$ ) to the graph $G\left[V^{\prime \prime}\right]$ to obtain a collection of $k-1$ path forests $P_{1}, \ldots, P_{k-1}$ which contain at most $n / \log ^{3} n$ paths each.

Our goal at this point is to connect all the paths belonging to $P_{1}$ together with $P_{Q}$ into the first cycle $C_{1}$, connect all the paths belonging to $P_{2}$ together with the absorber $H$ into the second cycle $C_{2}$, and the paths belonging to each $P_{i}$ for all $i \geqslant 3$ into a cycle $C_{i}$. Along the way of constructing the cycles $C_{2}, \ldots, C_{k-1}$, we may reuse some of the vertices of $Q_{A} \cup Q_{B}$, which is not a problem.

Set $\gamma^{\prime}:=\gamma_{1}^{2} / 8$. Let $m_{i}$ denote the number of paths in $P_{i}$, for all $i \in[k-1]$. Take an arbitrary ordering of the paths in each $P_{i}$ and denote their endpoints by $s_{i}^{j}$ and $t_{i}^{j}$, for all $i \in[k-1], j \in\left[m_{i}\right]$. We again artificially make every vertex either $A$-expanding or $B$-expanding depending on their degree into $U \cap A$ and $U \cap B$, keeping at least $\gamma^{\prime}|U| p$ edges for each vertex. This is possible due to (8) and our choice of $\gamma_{1}$. Next, we construct a set $\left\{u_{1}, \ldots, u_{x}\right\}$, for some $0 \leqslant x \leqslant 2 \mathrm{kn} / \log ^{3} n$, by sequentially choosing vertices from $Q_{A}$ or $Q_{B}$ depending on the difference in the number of $A$-pairs and $B$-pairs. It is easy to see that one can greedily choose such a set in order for

$$
\begin{aligned}
\mathcal{P}=\{ & \left\{v_{q}, s_{1}^{1}\right\},\left\{t_{1}^{j}, s_{1}^{j+1}\right\}_{j \in\left[m_{1}-1\right]},\left\{t_{1}^{m_{1}}, v_{1}\right\}, & & \text { (cycle } \left.C_{1} \text { including } P_{Q}\right) \\
& \left\{t_{i}^{j}, s_{i}^{j+1}\right\}_{3 \leqslant i \leqslant k-1, j \in\left[m_{i}-1\right]},\left\{t_{i}^{m_{i}}, s_{i}^{1}\right\}_{i \in[k-1]} & & \text { (cycles } \left.C_{3}, \ldots, C_{k-1}\right) \\
& \left.\left\{b, s_{2}^{1}\right\},\left\{t_{2}^{j}, s_{2}^{j+1}\right\}_{j \in\left[m_{2}-1\right]},\left\{t_{2}^{m_{2}}, u_{1}\right\},\left\{u_{i}, u_{i+1}\right\}_{i \in[x-1]},\left\{u_{x}, a\right\}\right\} & & \text { (cycle } \left.C_{2} \text { including } H\right)
\end{aligned}
$$

to be such that the number of $A$-pairs and $B$-pairs in $\mathcal{P}$ is the same.

Finally, we apply the Connecting Lemma (Lemma 3.4) to the graph obtained from $G$ by substituting $G[U]$ by $F[U]$, and with $\gamma^{\prime}$ (as $\gamma$ ), $V(G) \backslash U$ (as $U$ ), $U$ (as $W$ ), and the multigraph $M$ with the vertex set

$$
V(M)=\{a, b\} \cup\left\{s_{i}^{j}, t_{i}^{j}\right\}_{i \in[k-1], j \in\left[m_{i}\right]} \cup\left\{v_{i}\right\}_{i \in[q]}
$$


and the edge set $E(M)=\mathcal{P}$. This is indeed possible as $(i) F[U]$ is a $\gamma_{1} p$-expander and thus a $\gamma^{\prime} p$-expander as well, (ii) $|U|=\left\lfloor\varepsilon n /\left(C \log ^{2} n\right)\right\rfloor$ and $\beta \leqslant \eta n p / \log ^{3} n$, and (iii) all vertices have degree at least $\gamma^{\prime}|U| p$ into $U$ due to Claim 3.9 and (8). Therefore, we obtain $k-1$ cycles $C_{1}, C_{2}, \ldots, C_{k-1}$, where $C_{2}$ contains $P_{Q}$ as a subgraph and $C_{1}$ contains $H$ and possibly some vertices of $P_{Q}$ as a subgraph. As in the case $k=2$, replacing $H$ in $C_{1}$ by an absorbing $a b$-path $P^{\prime}$ using all the uncovered vertices of $U$ completes the proof.

In the remainder of the paper we supply the missing proofs of the lemmas stated above, each section fully being dedicated to one of the lemmas.

\section{The Inheritance Lemma}

For the convenience of the reader, we repeat the statement of Lemma 3.5.

Lemma 3.5 (Inheritance Lemma). For every $\gamma \in(0,1)$, there exist positive $\gamma_{1}(\gamma)$ and $C(\gamma)$ such that the following holds for all sufficiently large $n$. Let $p \in(0,1)$ and $\beta>0$, let $G$ be $a(p, \beta)$ sparse $\gamma p$-expander on $n$ vertices, and let $r$ be a positive integer such that $r p \geqslant C \cdot \max \{\log n, \beta\}$. Then the number of subsets $R \subseteq V(G)$ of size $r$ for which $G[R]$ is a $\gamma_{1} p$-expander is at least $\left(1-n^{-1}\right)\left(\begin{array}{l}n \\ r\end{array}\right)$.

Proof. Assume that $R \subseteq V(G)$ is a set of size $r$ chosen uniformly at random. We aim to show that $R$ induces an $\Omega(p)$-expander with probability at least $1-n^{-1}$ which in turn implies the lemma.

Note that as $G$ is a $\gamma p$-expander, it has minimum degree at least $\gamma p(n-1)$. Then, as $R$ is a uniformly random subset of size $r \geqslant C \log n / p$ standard application of Chernoff's inequality and the union bound shows that with probability at least $1-o\left(n^{-2}\right)$ (for $C$ large enough), we have

$$
\delta(G[R]) \geqslant \gamma r p / 2 .
$$

To show that $G[R]$ is a $\gamma_{1} p$-expander, we need to verify that for all partitions $X \cup Y=R$, we have $e(X, Y) \geqslant \gamma_{1} p|X||Y|$. We first show that if (10) holds, then this is indeed the case for all partitions where one of the parts, say $X$, is smaller than $\gamma r / 4$. For this, we use the fact that $G$, and hence also $G[R]$, is $(p, \beta)$-sparse. Applying Lemma 2.1 to the graph $G[R]$ and using $|X| \leqslant \gamma r / 4$ and (10), we obtain

$$
e(X, Y) \geqslant \gamma|X| r p / 4-\beta|X| .
$$

By choosing $C$ to be sufficiently large and from the assumption that $\beta \leqslant r p / C$ we further get

$$
e(X, Y) \geqslant \gamma|X| r p / 4-\beta|X| \geqslant \gamma|X| r p / 5 \geqslant \gamma|X||Y| p / 5 .
$$

In particular, the expansion property holds with $\gamma_{1}=\gamma / 5$.

The argument for the remaining case is more complicated and can be summarised as follows. We apply the sparse regularity lemma to the graph $G$, thus obtaining an $(\varepsilon, \tilde{p})$-regular partition $V_{0} \cup V_{1} \cup \ldots \cup V_{t}$, where $\varepsilon$ is a small positive constant and $\tilde{p}=e(G) /\left(\begin{array}{l}n \\ 2\end{array}\right)$ is the density of $G$. The random set $R$ intersects each part of the regular partition in roughly the expected number of vertices. Moreover, whenever $\left(V_{i}, V_{j}\right)$ is an $(\varepsilon, \tilde{p})$-regular pair with density $d_{i j} \tilde{p}$, then the pair $\left(V_{i} \cap R, V_{j} \cap R\right)$ is $\left(\varepsilon^{\prime}, d_{i j} \tilde{p}\right)$-lower-regular with a slightly weaker parameter $\varepsilon^{\prime}$, which follows from Lemma 2.6. Now if we have a partition $X \cup Y=R$, where $|X|,|Y| \geqslant \gamma r / 4$, then we 'approximate' it by a partition $X^{\prime} \cup Y^{\prime}=V(G) \backslash V_{0}$ by letting $X^{\prime}$ (resp. $Y^{\prime}$ ) be the union of the classes in the regular partition that intersect $X$ (resp. $Y$ ) in some $\Omega(r)$ vertices (note that the sets defined in this way may not be disjoint; there is thus a simple cleaning-up step to make sure that this is the case). Since $G$ is a $\gamma p$-expander and $V_{0}$ is small, we know that there are $\gamma p\left|X^{\prime}\right|\left|Y^{\prime}\right|$ edges in $G\left[X^{\prime}, Y^{\prime}\right]$, which implies that there are many dense regular pairs $\left(V_{i}, V_{j}\right)$ such that $V_{i} \subseteq X^{\prime}$ and $V_{j} \subseteq Y^{\prime}$. Because for such pairs the pair $\left(V_{i} \cap R, V_{j} \cap R\right)$ is lower-regular, $\left(V_{i} \cap X, V_{j} \cap Y\right)$ contains many edges, that is, there are many edges in $G[X, Y]$. We now give the details. 
Set $\delta=1 / e$ and $\tilde{p}=e(G) /\left(\begin{array}{l}n \\ 2\end{array}\right)$, and let $d$ and $\varepsilon^{\prime}<d / 2$ be sufficiently smaller than $\gamma$ in order to support the arguments that follow. Let $\varepsilon_{0}=\varepsilon_{0}{ }_{2.6}\left(\varepsilon^{\prime}, \delta\right)$ and $D=D_{2.6}\left(\varepsilon^{\prime}\right)$. Lastly, let $\varepsilon$ be sufficiently small with respect to all previously chosen constants, let $t$ be as given by Lemma 2.5 applied for $\varepsilon$, and assume that $C$ is large enough (in particular, $C$ is much larger than $t$ ). For ease of reference we point out that we have the following hierarchy of constants

$$
0<\varepsilon \ll \varepsilon_{0} \ll \varepsilon^{\prime} \ll d \ll \gamma<1 \ll t \ll C .
$$

Observe that $G$ being a $\gamma p$-expander which is $(p, \beta)$-sparse implies $\gamma p / 2 \leqslant \tilde{p} \leqslant 4 p$.

We apply the sparse regularity lemma (Lemma 2.5) to $G$ for $\varepsilon$ to obtain an $(\varepsilon, \tilde{p}$ )-regular partition $\left(V_{i}\right)_{i=0}^{t}$ of $V(G)$, where $V_{0}$ is the exceptional class of size $\left|V_{0}\right| \leqslant \varepsilon n$. Since $\left|V_{1}\right|=\cdots=$ $\left|V_{t}\right|$, we have $(1-\varepsilon) n / t \leqslant\left|V_{i}\right| \leqslant n / t$ for all $i \in[t]$.

For each $0 \leqslant i \leqslant t$, set $U_{i}:=R \cap V_{i}$. Note that each $U_{i}$ is a random subset of $V_{i}$ whose size follows a hypergeometric distribution with mean $r\left|V_{i}\right| / n \geqslant C \log n \cdot \min \{\varepsilon,(1-\varepsilon) / t\}$. By our choice of $C$, Chernoff's inequality together with the union bound shows that with probability at least $1-o\left(n^{-2}\right)$, all sets $U_{i}, i \in[t]$, satisfy $\left|U_{i}\right|=(1 \pm \varepsilon) r\left|V_{i}\right| / n$ and similarly $\left|U_{0}\right| \leqslant(1+\varepsilon) \varepsilon r$. Recalling that $(1-\varepsilon) n / t \leqslant\left|V_{i}\right| \leqslant n / t$, this implies in particular $\left|U_{i}\right|=\left(1 \pm \varepsilon^{\prime}\right) r / t$ and $\left|U_{0}\right| \leqslant \varepsilon^{\prime} r$.

Consider now a fixed $(\varepsilon, \tilde{p})$-regular pair $\left(V_{i}, V_{j}\right)$ with density at least $d \tilde{p}$. By definition, $\left(V_{i}, V_{j}\right)$ is then also $(\varepsilon / d, d \tilde{p})$-lower-regular. We apply Lemma 2.6 with $\varepsilon^{\prime}, \delta, d \tilde{p}$ (as $p$ ), and $\left|U_{i}\right|,\left|U_{j}\right|$ (as $\left.q_{1}, q_{2}\right)$ to get that with probability at least

$$
1-\delta^{\min \left\{q_{1}, q_{2}\right\}}=1-\delta^{\left(1-\varepsilon^{\prime}\right) C \log n / t} \geqslant 1-o\left(n^{-2}\right),
$$

the pair $\left(U_{i}, U_{j}\right)$ is $\left(\varepsilon^{\prime}, d \tilde{p}\right)$-lower-regular. Indeed, we can apply the lemma since $\left|U_{i}\right|,\left|U_{j}\right| \geqslant$ $\left(1-\varepsilon^{\prime}\right) r / t \geqslant D(d \tilde{p})^{-1}$ due to the assumption of the lemma on $r$ and the fact that $\gamma \tilde{p} / 2 \leqslant p$, and $\varepsilon / d<\varepsilon_{0}$. Therefore, the union bound over at most $t^{2}$ pairs $\left(V_{i}, V_{j}\right)$ shows that with probability at least $1-o\left(n^{-2}\right)$, the following two properties hold:

(i) $\left|U_{0}\right| \leqslant \varepsilon^{\prime} r$ and $\left(1-\varepsilon^{\prime}\right) r / t \leqslant(1-\varepsilon) r\left|V_{i}\right| / n \leqslant\left|U_{i}\right| \leqslant\left(1+\varepsilon^{\prime}\right) r / t$, for all $i \in[t]$, and

(ii) whenever $\left(V_{i}, V_{j}\right)$ is an $(\varepsilon, \tilde{p})$-regular pair with density $d_{i j} \tilde{p} \geqslant d \tilde{p}$, then $\left(U_{i}, U_{j}\right)$ is $\left(\varepsilon^{\prime}, d_{i j} \tilde{p}\right)$ lower-regular.

From these two properties we show the expansion of $G[R]$ deterministically for all partitions $X \cup Y=R$ where $|X|,|Y| \geqslant \gamma r / 4$. Let us fix such a partition and assume without loss of generality that $|X| \leqslant r / 2 \leqslant|Y|$. As outlined above, we approximate the given partition of $R$ by a certain partition of the vertices of $V(G) \backslash V_{0}$, or, to be more precise, by a partition of the classes $V_{1}, \ldots, V_{t}$. We now describe precisely how to do this.

Let $\mathcal{I}_{X}=\left\{i \in[t]:\left|U_{i} \cap X\right| \geqslant \gamma\left|U_{i}\right| / 10\right\}$ and $\mathcal{I}_{Y}=\left\{i \in[t]:\left|U_{i} \cap Y\right| \geqslant \gamma\left|U_{i}\right| / 10\right\}$. Note that $\mathcal{I}_{X} \cup \mathcal{I}_{Y}=[t]$, although $\mathcal{I}_{X}$ and $\mathcal{I}_{Y}$ are not necessarily disjoint. Using $(i)$ we have

$$
\left|\mathcal{I}_{X}\right| \cdot\left(1+\varepsilon^{\prime}\right) r / t \geqslant \sum_{i \in \mathcal{I}_{X}}\left|U_{i}\right| \geqslant|X|-\sum_{i \notin \mathcal{I}_{X}} \gamma\left|U_{i}\right| / 10-\left|U_{0}\right| \geqslant \gamma r / 4-\left(1+\varepsilon^{\prime}\right) \gamma r / 10-\varepsilon^{\prime} r
$$

and hence

$$
\left|\mathcal{I}_{X}\right| \geqslant \frac{\gamma r / 4-\left(1+\varepsilon^{\prime}\right) \gamma r / 10-\varepsilon^{\prime} r}{\left(1+\varepsilon^{\prime}\right) r / t} \geqslant \gamma t / 8,
$$

using the fact that $\varepsilon^{\prime}$ is sufficiently small compared to $\gamma$. In the same way, using $|Y| \geqslant r / 2$, we also obtain $\left|\mathcal{I}_{Y}\right| \geqslant t / 4$. Since $\mathcal{I}_{X} \cup \mathcal{I}_{Y}=[t]$, it follows that there exists a partition $\mathcal{J}_{X} \cup \mathcal{J}_{Y}=[t]$ such that $\mathcal{J}_{X} \subseteq \mathcal{I}_{X}$ and $\mathcal{J}_{Y} \subseteq \mathcal{I}_{Y}$ and such that $\left|\mathcal{J}_{X}\right| \geqslant \gamma t / 8$ and $\left|\mathcal{J}_{Y}\right| \geqslant t / 4$.

Next, we say that a pair $\left(V_{i}, V_{j}\right)$ for $1 \leqslant i<j \leqslant t$ is good if it is $(\varepsilon, \tilde{p})$-regular with density $d_{i j} \tilde{p} \geqslant d \tilde{p}$, and if $(i, j) \in \mathcal{J}_{X} \times \mathcal{J}_{Y}$. From (ii) it follows that if $\left(V_{i}, V_{j}\right)$ is good, then $\left(U_{i}, U_{j}\right)$ is $\left(\varepsilon^{\prime}, d_{i j} \tilde{p}\right)$-lower-regular. The lower-regularity and $\left|U_{i} \cap X\right| \geqslant \gamma\left|U_{i}\right| / 10 \geqslant \varepsilon^{\prime}\left|U_{i}\right|$ and $\left|U_{j} \cap Y\right| \geqslant$ $\gamma\left|U_{j}\right| / 10 \geqslant \varepsilon^{\prime}\left|U_{j}\right|$ in turn imply

$$
e\left(U_{i} \cap X, U_{j} \cap Y\right) \geqslant\left(1-\varepsilon^{\prime}\right) d_{i j} \tilde{p}\left|U_{i} \cap X\right|\left|U_{j} \cap Y\right| \geqslant \frac{\gamma^{2}}{100} \cdot\left(1-\varepsilon^{\prime}\right) d_{i j} \tilde{p}\left|U_{i}\right|\left|U_{j}\right| .
$$


Together with $(i)$, we obtain

$$
d_{i j} \tilde{p}\left|U_{i}\right|\left|U_{j}\right| \geqslant\left(1-\varepsilon^{\prime}\right)^{3} d_{i j} \tilde{p}\left|V_{i}\right|\left|V_{j}\right| r^{2} / n^{2} \geqslant e\left(V_{i}, V_{j}\right) r^{2} /\left(2 n^{2}\right),
$$

which then implies $e\left(U_{i} \cap X, U_{j} \cap Y\right) \geqslant \gamma^{2} e\left(V_{i}, V_{j}\right) r^{2} /\left(200 n^{2}\right)$. In particular,

$$
e(X, Y) \geqslant \sum_{\substack{1 \leqslant i<j \leqslant t \\\left(V_{i}, V_{j}\right) \text { good }}} e\left(U_{i} \cap X, U_{j} \cap Y\right) \geqslant \frac{\gamma^{2} r^{2}}{200 n^{2}} \sum_{\substack{1 \leqslant i<j \leqslant t \\\left(V_{i}, V_{j}\right) \text { good }}} e\left(V_{i}, V_{j}\right) .
$$

To complete the proof, we show that many edges of $G$ go between good pairs $\left(V_{i}, V_{j}\right)$. Set $X^{\prime}=\bigcup_{i \in \mathcal{J}_{X}} V_{i}$ and $Y^{\prime}=\bigcup_{i \in \mathcal{J}_{Y}} V_{i}$. Note that $X^{\prime} \cup Y^{\prime}=V(G) \backslash V_{0}$. Since $\left|\mathcal{J}_{X}\right| \geqslant \gamma t / 8$ and $\left|V_{i}\right| \geqslant(1-\varepsilon) n / t$, we have $\left|X^{\prime}\right| \geqslant \gamma n / 10$, for $\varepsilon$ small enough. Similarly, $\left|\mathcal{J}_{Y}\right| \geqslant t / 4$ implies $\left|Y^{\prime}\right| \geqslant n / 5$. From the assumption that $G$ is a $\gamma p$-expander, we obtain

$$
e\left(X^{\prime}, Y^{\prime} \cup V_{0}\right) \geqslant \gamma p\left|X^{\prime}\right|\left|Y^{\prime}\right| \geqslant \gamma^{2} n^{2} p / 50 .
$$

On the other hand, we have

$$
e\left(V_{0}, X^{\prime}\right) \leqslant p\left|V_{0}\right|\left|X^{\prime}\right|+\beta \sqrt{\left|V_{0}\right|\left|X^{\prime}\right|} \leqslant 2 \varepsilon n^{2} p .
$$

where we used $\left|V_{0}\right| \leqslant \varepsilon n$ and $\beta \leqslant r p / C \leqslant n p / C \leqslant \varepsilon n p$ (provided $C$ is large enough). From these it follows that

$$
e\left(X^{\prime}, Y^{\prime}\right) \geqslant e\left(X^{\prime}, Y^{\prime} \cup V_{0}\right)-2 \varepsilon n^{2} p \geqslant \gamma^{2} n^{2} p / 100,
$$

using that $\varepsilon$ is sufficiently small compared to $\gamma$.

Using once more the fact that $G$ is $(p, \beta)$-sparse, we have $e\left(V_{i}, V_{j}\right) \leqslant\left|V_{i}\right|\left|V_{j}\right| p+\beta \sqrt{\left|V_{i}\right|\left|V_{j}\right|} \leqslant$ $2 n^{2} p / t^{2}$ for all $i, j \in[t]$. By the definition of an $(\varepsilon, \tilde{p})$-regular partition, there are at most $\varepsilon t^{2}$ pairs $\left(V_{i}, V_{j}\right)$ that are not $(\varepsilon, \tilde{p})$-regular. Hence, the number of edges in $G\left[X^{\prime}, Y^{\prime}\right]$ which go between non-regular pairs $\left(V_{i}, V_{j}\right)$ is at most $2 \varepsilon n^{2} p$. Moreover, the number of edges in $G\left[X^{\prime}, Y^{\prime}\right]$ with one endpoint in each part of a pair $\left(V_{i}, V_{j}\right)$ with density below $d \tilde{p}$ is clearly at most $d n^{2} \tilde{p} \leqslant 4 d n^{2} p$. Lastly, note that by definition of $X^{\prime}$ and $Y^{\prime}$, for every edge $e \in G\left[X^{\prime}, Y^{\prime}\right]$, there is some $\left(V_{i}, V_{j}\right)$ with $(i, j) \in \mathcal{J}_{X} \times \mathcal{J}_{Y}$ such that $e \in G\left[V_{i}, V_{j}\right]$. Then (12) and the definition of a good pair give

$$
\sum_{\substack{1 \leqslant i<j \leqslant t \\\left(V_{i}, V_{j}\right) \text { good }}} e\left(V_{i}, V_{j}\right) \geqslant e\left(X^{\prime}, Y^{\prime}\right)-2 \varepsilon n^{2} \tilde{p}-4 d n^{2} p \geqslant \gamma^{2} n^{2} p / 200,
$$

using again that $\varepsilon, \varepsilon^{\prime}$, and $d$ are small compared to $\gamma$. Finally, with (11), we get

$$
e(X, Y) \geqslant \frac{\gamma^{2} r^{2}}{200 n^{2}} \sum_{\substack{1 \leqslant i<j \leqslant t \\\left(V_{i}, V_{j}\right) \text { good }}} e\left(V_{i}, V_{j}\right) \geqslant \frac{\gamma^{4}}{200^{2}} \cdot r^{2} p \geqslant \gamma_{1}|X||Y| p,
$$

for $\gamma_{1}=\gamma^{4} / 40000$.

\section{The Connecting Lemma}

The main result of this section is essentially that if $G$ is a pseudorandom graph and $W$ is a sufficiently large subset inducing a good expander, then $G$ is 'highly connected via $W$ ', i.e., it is possible to connect many given pairs of vertices using short paths whose internal vertices lie entirely in $W$. We formally restate the lemma for the convenience of the reader.

Lemma 3.4 (Connecting Lemma). For every $\gamma \in(0,1)$ and $\Delta>0$, there exists a positive $C(\gamma, \Delta)$ such that the following holds for all sufficiently large $n$. Let $p \in(0,1)$ and $\beta>0$, let $G$ be a $(p, \beta)$-sparse graph on $n$ vertices, and let $U, W \subseteq V(G)$ be disjoint subsets such that:

(i) $G[W]$ is a $\gamma p$-expander,

(ii) $|W| \geqslant C \beta \sqrt{\log n} / p$, and

(iii) every vertex $u \in U$ satisfies $\operatorname{deg}(u, W) \geqslant \gamma|W| p$. 
Let $\ell=\lceil 30 \log n /(\gamma \log \log n)\rceil$. Then for every multigraph $M$ with $V(M) \subseteq U$, at most $|W| /(C \ell)$ edges, and maximum degree at most $\Delta$, there exists an $(M, W, \ell)$-matching in $G$.

The main step towards proving the Connecting Lemma is to prove the following technical auxiliary lemma whose proof we defer to the end of the section.

Lemma 5.1. For every $\gamma \in(0,1)$, the following holds for all sufficiently large $n$, all $p \in(0,1)$, and all $\beta>0$. Let $G$ be $a(p, \beta)$-sparse graph on $n$ vertices and let $U, W \subseteq V(G)$ be disjoint subsets such that:

(i) $G[W]$ is a $\gamma p$-expander,

(ii) $|W| \geqslant 10 \gamma^{-1} \beta \sqrt{\log n} / p$, and

(iii) every vertex $u \in U$ satisfies $\operatorname{deg}(u, W) \geqslant \gamma|W| p$.

Let $\ell=\lceil 10 \log n /(\gamma \log \log n)\rceil$. Then for every subset $Z \subseteq W$ with

$$
|Z| \leqslant \min \{\gamma|W| / 20,|U| \log n\},
$$

there is a vertex $x \in U$ such that $\left|N^{\ell}(x, W \backslash Z)\right|>|W| / 2$.

Proof of Lemma 3.4. We define an $\ell$-uniform hypergraph $\mathcal{H}$ on the vertex set $E(M) \cup W$, where for every edge $e \in E(M)$ and every set $Y \subseteq W$ of size $\ell-1$, we add a hyperedge $\{e\} \cup Y$ if and only if $G$ contains a path joining the endpoints of $e$ whose internal vertices belong to $Y$. Hence, if there is an $E(M)$-saturating matching in $\mathcal{H}$, then $G$ contains an $(M, W, \ell)$-matching. We use Haxell's criterion (Theorem 2.4) to show that $\mathcal{H}$ contains an $E(M)$-saturating matching.

For this, let $S \subseteq E(M)$ and $Z \subseteq W$ be subsets with $|Z| \leqslant 2 \ell|S|$. Since $M$ has maximum degree at most $\Delta$, we can greedily find a subset $S^{\prime} \subseteq S$ of size $|S| /(2 \Delta)$ such that the edges in $S^{\prime}$ are pairwise disjoint. In other words, there exist disjoint sets $U_{1}, U_{2} \subseteq U$ of size $\left|U_{1}\right|=\left|U_{2}\right| \geqslant$ $|S| /(2 \Delta)$, and a bijection $\varphi: U_{1} \rightarrow U_{2}$, such that for every $u \in U_{1}$, the pair $\{u, \varphi(u)\}$ belongs to $S^{\prime}$. It is enough to show that for some $u \in U_{1}, G$ contains a $u \varphi(u)$-path of length at most $\ell$ whose internal vertices are all in the set $W \backslash Z$. Indeed, this implies that $\mathcal{H}$ contains an edge intersecting $S^{\prime}$ (and hence $S$ ) and not intersecting $Z$, showing that Haxell's criterion is satisfied. In the remainder we show that such a vertex $u \in U_{1}$ exists.

Let $\ell_{1}=\lceil 10 \log n /(\gamma \log \log n)\rceil$ and let $U_{1}^{\prime} \subseteq U_{1}$ be the set consisting of all vertices $u \in U_{1}$ for which $\left|N^{\ell_{1}}(u, W \backslash Z)\right|>|W| / 2$. We claim that $\left|U_{1}^{\prime}\right|>\left|U_{1}\right| / 2$. Towards a contradiction suppose $\left|U_{1}^{\prime}\right| \leqslant\left|U_{1}\right| / 2$ and let $U_{1}^{\prime \prime}=U_{1} \backslash U_{1}^{\prime}$. Note, $\left|U_{1}^{\prime \prime}\right| \geqslant\left|U_{1}\right| / 2 \geqslant|S| /(4 \Delta)$. Since $|Z| \leqslant 2 \ell|S| \leqslant$ $\left|U_{1}^{\prime \prime}\right| \log n$ and also $|Z| \leqslant 2 \ell|S| \leqslant 2 \ell e(M) \leqslant 2|W| / C$, we have

$$
|Z| \leqslant \min \left\{\gamma|W| / 20,\left|U_{1}^{\prime \prime}\right| \log n\right\}
$$

for sufficiently large $C$. By the assumption $|W| \geqslant C \beta \sqrt{\log n} / p$, we also see that $|W| \geqslant$ $10 \gamma^{-1} \beta \sqrt{\log n} / p$. Therefore, by Lemma 5.1 applied for $U_{1}^{\prime \prime}$ (as $U$ ), $W, Z$, and $\ell_{1}$ (as $\ell$ ), there exists a vertex $u \in U_{1}^{\prime \prime}$ such that $\left|N^{\ell_{1}}(u, W \backslash Z)\right|>|W| / 2$, which is a contradiction with the definition of $U_{1}^{\prime \prime}$. It follows that, indeed, $\left|U_{1}^{\prime}\right|>\left|U_{1}\right| / 2$.

Analogously, the set $U_{2}^{\prime} \subseteq U_{2}$ of all vertices $u \in U_{2}$ for which $\left|N^{\ell_{1}}(u, W \backslash Z)\right|>|W| / 2$ has size $\left|U_{2}^{\prime}\right|>\left|U_{2}\right| / 2$. It then follows that there is some $u \in U_{1}^{\prime}$ such that $\varphi(u) \in U_{2}^{\prime}$. Thus, from both $u$ and $\varphi(u)$ it is possible to reach strictly more than $|W| / 2$ vertices of $W \backslash Z$ via paths of length at most $\ell_{1}$ whose internal vertices belong to $W \backslash Z$. Hence, there must exist a $u \varphi(u)$-path of length at most $2 \ell_{1} \leqslant \ell$ whose internal vertices all lie in $W \backslash Z$. This completes the proof.

We now proceed with the proof of Lemma 5.1.

Proof of Lemma 5.1. Without loss of generality, assume $|U| \leqslant|W| /(2 \log n)$. Indeed, if $U$ contains more than $|W| /(2 \log n)$ elements, then replacing it by a subset of size $\lfloor|W| /(2 \log n)\rfloor$ does not violate any of the assumptions $(i)-($ iii $)$ nor the bound on the size of $Z$. 
We first prove an auxiliary claim about expansion of certain subsets which we use in the proof of the lemma.

Claim 5.2. If $X \subseteq U \cup W$ is such that $|X| \geqslant|U|$ and $e(X, W \backslash X) \geqslant \gamma|X||W| p / 2$, then

$$
|N(X, W \backslash(X \cup Z))| \geqslant \min \{|X| \log n, \gamma|W| / 5\} .
$$

Proof. Let us denote $N(X, W \backslash X)$ by $Y$. Recall that since $G$ is $(p, \beta)$-sparse, we have

$$
e(X, W \backslash X)=e(X, Y) \leqslant|X||Y| p+\beta \sqrt{|X||Y|} .
$$

Combining this with the assumption $e(X, W \backslash X) \geqslant \gamma|X||W| p / 2$, we obtain

$$
|Y| \geqslant \frac{\gamma|W|}{2}-\frac{\beta \sqrt{|Y|}}{\sqrt{|X| p}}
$$

If $\sqrt{|Y|} \leqslant \gamma \sqrt{|X|}|W| p /(4 \beta)$, then the above inequality gives $|Y| \geqslant \gamma|W| / 4$. In this case, it follows from the assumption $|Z| \leqslant \gamma|W| / 20$ that

$$
|N(X, W \backslash(X \cup Z))| \geqslant \gamma|W| / 4-|Z| \geqslant \gamma|W| / 5 .
$$

On the other hand, if $\sqrt{|Y|}>\gamma \sqrt{|X|}|W| p /(4 \beta)$ then using the assumption $|W| \geqslant 10 \gamma^{-1} \beta \sqrt{\log n} / p$ we get the lower bound $\sqrt{|Y|} \geqslant 2 \sqrt{|X| \log n}$, or, equivalently, $|Y| \geqslant 4|X| \log n$. Since $|Z| \leqslant$ $|U| \log n \leqslant|X| \log n$ we obtain

$$
|N(X, W \backslash(X \cup Z))| \geqslant 4|X| \log n-|Z| \geqslant|X| \log n,
$$

completing the proof of the claim.

We are now ready to prove the lemma. For every integer $j \geqslant 0$, set

$$
\ell_{j}:=\lceil\log n /(\gamma \log \log n)\rceil+(j+1)\lceil 5 / \gamma\rceil .
$$

The goal is to construct a sequence of subsets $U=U_{0} \supseteq U_{1} \supseteq U_{2} \supseteq \cdots$ such that for every $j \geqslant 0$ we have $\left|U_{j+1}\right| \leqslant\left\lceil\left|U_{j}\right| / \log n\right\rceil$ and

$$
\left|N^{\ell_{j}}\left(U_{j}, W \backslash Z\right)\right|>|W| / 2 .
$$

Note that $\left|U_{j}\right| \leqslant\left\lceil\left|U_{j-1}\right| / \log n\right\rceil$ implies that either $\left|U_{j}\right|=1$ or $\left|U_{j}\right| \leqslant 2\left|U_{j-1}\right| / \log n$, and therefore, for $j=\lceil\log n /(\log \log n-\log 2)\rceil$, we have $\left|U_{j}\right|=1$. Moreover, for this $j$, we have $\ell_{j} \leqslant 10 \log n /(\gamma \log \log n) \leqslant \ell$. In this way, the statement of the lemma follows, provided we can indeed construct the sets $U_{0}, U_{1}, U_{2}, \ldots$ with the properties mentioned above.

For the set $U_{0}=U$, we only need to verify that $\left|N^{\ell_{0}}\left(U_{0}, W \backslash Z\right)\right|>|W| / 2$. Note that as $e\left(U_{0}, W \backslash U_{0}\right) \geqslant \gamma\left|U_{0}\right||W| p$ due to (iii), Claim 5.2 for $U_{0}$ (as $X$ ) implies

$$
\left|N^{1}\left(U_{0}, W \backslash Z\right)\right| \geqslant \min \{|U| \log n, \gamma|W| / 5\} \geqslant|U|,
$$

where the last inequality holds because of our assumption $|U| \leqslant|W| /(2 \log n)$. Moreover, if for some $i \geqslant 1$, we have $|U| \leqslant\left|N^{i}\left(U_{0}, W \backslash Z\right)\right| \leqslant|W| / 2$, then as

$$
\begin{aligned}
& e\left(N^{i}\left(U_{0}, W \backslash Z\right), W \backslash N^{i}\left(U_{0}, W \backslash Z\right)\right) \stackrel{(i)}{\geqslant} \gamma\left|N^{i}\left(U_{0}, W \backslash Z\right)\right|\left|W \backslash N^{i}\left(U_{0}, W \backslash Z\right)\right| p \\
& \geqslant \gamma\left|N^{i}\left(U_{0}, W \backslash Z\right)\right||W| p / 2,
\end{aligned}
$$

Claim 5.2 applied to $N^{i}\left(U_{0}, W \backslash Z\right)$ (as $\left.X\right)$ shows

$$
\left|N^{i+1}\left(U_{0}, W \backslash Z\right)\right| \geqslant\left|N^{i}\left(U_{0}, W \backslash Z\right)\right|+\min \left\{|U| \log ^{i+1} n, \gamma|W| / 5\right\} .
$$

One easily sees that for $\ell_{0}=\lceil\log n /(\gamma \log \log n)\rceil+\lceil 5 / \gamma\rceil$, we have $\left|N^{\ell_{0}}\left(U_{0}, W \backslash Z\right)\right|>|W| / 2$ as required.

Suppose now we have constructed the set $U_{j}$ as above and want to construct $U_{j+1}$. We thus assume

$$
\left|N^{\ell_{j}}\left(U_{j}, W \backslash Z\right)\right|>|W| / 2 .
$$


By averaging, there exists a subset $U_{j+1} \subseteq U_{j}$ of size $\left|U_{j+1}\right| \leqslant\left\lceil\left|U_{j}\right| / \log n\right\rceil$ such that

$$
\left|N^{\ell_{j}}\left(U_{j+1}, W \backslash Z\right)\right|>|W| /(2 \log n) \geqslant|U| .
$$

Successively applying Claim 5.2 at most $[5 / \gamma\rceil$ times, it is easy to see that

$$
\left|N^{\ell_{j}+[5 / \gamma]}\left(U_{j+1}, W \backslash Z\right)\right|>|W| / 2 .
$$

Indeed, in a single step the set $N^{\ell_{j}}\left(U_{j+1}, W \backslash Z\right)$ expands to a set of size

$$
\min \{|W| /(2 \log n) \cdot \log n, \gamma|W| / 5\} \geqslant \gamma|W| / 5,
$$

and in at most $[5 / \gamma]-1$ additional steps, it expands to a set of size greater than $|W| / 2$. This completes the proof of the lemma.

\section{The Absorber Lemma}

This section is dedicated to the construction of the absorbers and the proof of the Absorbing Lemma (Lemma 3.7). We recall the definition of an absorber first.

Definition 3.6 $((X, Y)$-absorber $)$. Let $X$ and $Y$ be disjoint sets of vertices. An $(X, Y)$-absorber is a graph $H$ with two designated vertices $a$ and $b$ (called the endpoints of the absorber) such that $X \cup Y \subseteq V(H) \backslash\{a, b\}$ and such that, for all subsets $X^{\prime} \subseteq X$ and $Y^{\prime} \subseteq Y$ with $\left|X^{\prime}\right|=\left|Y^{\prime}\right|$, $H$ contains an $a b$-path $P$ with $V(P)=V(H) \backslash\left(X^{\prime} \cup Y^{\prime}\right)$ (i.e., an $a b$-path using all vertices of $H$ with the exception of exactly the vertices in $\left.X^{\prime} \cup Y^{\prime}\right)$.

The most 'natural' way to construct an absorber would be to first find structures which 'absorb' one vertex of $X \cup Y$ at a time, and then depending on the sets $X^{\prime}$ and $Y^{\prime}$ individually decide which vertex needs to be 'absorbed'. Unfortunately, this is not possible in our case for the following reason. A single-vertex absorber $A_{x}$ would need to have two $a b$-paths: one containing $x$, one not containing $x$, and both containing all other vertices of $A_{x}$. It is an easy observation that such a structure necessarily contains an odd cycle. Since the graph $G$ might be bipartite, we cannot hope to find a single-vertex absorber described above in it.

In order to circumvent this we instead first build a collection of two-vertex absorbers $H_{x y}$, each of which contains two paths between its endpoints, one containing all the vertices of $H_{x y}$ including $x$ and $y$ and the other containing all vertices of $H_{x y}$ except $x$ and $y$. Such an absorber is depicted in Figure 1.

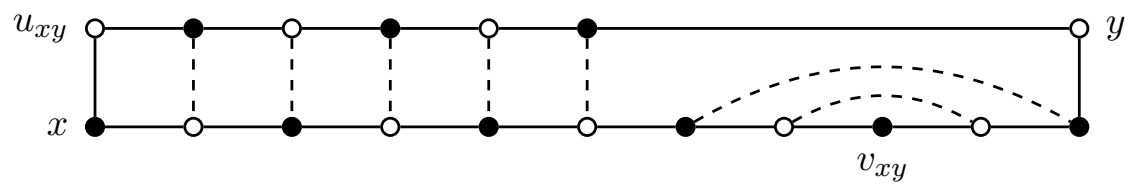

(A) An absorber $H_{x y}$ of unrealistically small size.

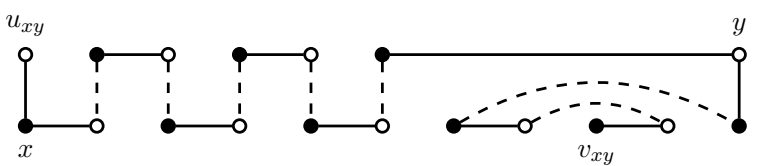

(в) The 'absorbing' $u_{x y} v_{x y}$-path

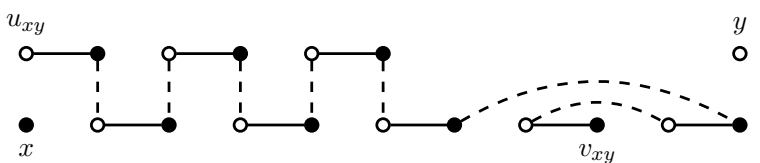

(c) The 'non-absorbing' $u_{x y} v_{x y}$-path.

FiguRE 1. An example of a two-vertex absorber for $x$ and $y$. The colours of the vertices correspond to the colour classes of the bipartite graph $G$. Dashed lines represent disjoint paths of length at most $\ell$ (see the proof below) and solid lines the actual edges. 
The following lemma provides us with a 'template' that we use to combine several two-vertex absorbers into an actual absorber. It is similar to a lemma of Montgomery [28, Lemma 10.7], which is proven in nearly the same way.

Lemma 6.1. There is an integer $n_{0} \in \mathbb{N}$ such that, for every $n \geqslant n_{0}$, there exist a bipartite graph $G=(A, B, E)$ with $|A|=|B|=2 n$ and $\Delta(G) \leqslant 40$, as well as subsets $A^{\prime} \subseteq A$ and $B^{\prime} \subseteq B$ with $\left|A^{\prime}\right|=\left|B^{\prime}\right|=n$, satisfying the following. For every set $Z \subseteq A^{\prime} \cup B^{\prime}$ with $\left|Z \cap A^{\prime}\right|=\left|Z \cap B^{\prime}\right|$, the graph $G[V(G) \backslash Z]$ contains a perfect matching.

Proof. Fix disjoint sets $A_{1}$ and $B_{1}$ with $\left|A_{1}\right|=\left|B_{1}\right|=n$. Let $H$ be a random bipartite graph with parts $A_{1}$ and $B_{1}$ obtained by inserting 20 independent random perfect matchings between $A_{1}$ and $B_{1}$ (and merging eventual multiple edges). We first show that w.h.p. $H$ satisfies the following properties:

(i) for every $X \subseteq A_{1}$ with $|X| \leqslant n / 2$, we have $\left|N_{H}(X)\right| \geqslant 2|X|$,

(ii) for every $Y \subseteq B_{1}$ with $|Y| \leqslant n / 2$, we have $\left|N_{H}(Y)\right| \geqslant 2|Y|$, and

(iii) for any two subsets $X \subseteq A_{1}$ and $Y \subseteq B_{1}$ with $|X|=|Y|=\lceil n / 4\rceil$, we have $e_{H}(X, Y)>0$.

Given two sets $X \subseteq A_{1}$ and $Y \subseteq B_{1}$, the probability that $N_{M}(X) \subseteq Y$ in a random matching $M$ is $\left(\begin{array}{c}|Y| \\ |X|\end{array}\right)\left(\begin{array}{c}n \\ |X|\end{array}\right)^{-1}$. Therefore, for a fixed integer $t \in[n / 4]$, the probability that there is a set $X \subseteq A_{1}$ with $|X|=t$ and $\left|N_{H}(X)\right| \leqslant 2 t$ is at most

$$
\left(\begin{array}{c}
n \\
t
\end{array}\right)\left(\begin{array}{c}
n \\
2 t
\end{array}\right)\left(\left(\begin{array}{c}
2 t \\
t
\end{array}\right)\left(\begin{array}{c}
n \\
t
\end{array}\right)^{-1}\right)^{20} \leqslant\left(\frac{e n}{t}\right)^{t}\left(\frac{e n}{2 t}\right)^{2 t}\left(\frac{2 t}{n}\right)^{20 t}=\left(2 e^{3}\left(\frac{2 t}{n}\right)^{17}\right)^{t}
$$

By a union bound over all $t \in[n / 4]$, and looking separately at the cases $t \leqslant \log n$ and $\log n \leqslant$ $t \leqslant n / 4$, the probability that $(i)$ fails tends to 0 as $n \rightarrow \infty$. In the same way, exchanging the roles of $A_{1}$ and $B_{1}$, one can show that w.h.p. the statement in (ii) holds as well. Lastly, the probability that (iii) fails is similarly at most

$$
\left(\begin{array}{c}
n \\
n / 4
\end{array}\right)^{2}\left(\left(\begin{array}{c}
3 n / 4 \\
n / 4
\end{array}\right)\left(\begin{array}{c}
n \\
n / 4
\end{array}\right)^{-1}\right)^{20} \leqslant(4 e)^{n / 2}\left(\frac{3}{4}\right)^{20 n / 4} \leqslant 2^{-n / 4}
$$

Thus, w.h.p. (iii) holds.

We now take any graph $H$ as above that satisfies $(i),(i i)$, and (iii) - such a graph exists for all large enough $n$ - and define $G$ by duplicating the vertices in $A_{1}$ and $B_{1}$ and keeping the edges as in $H$, except that a single edge of $H$ now corresponds to four edges in $G$. More precisely, we define $A=\left(A_{1} \times\{0\}\right) \cup\left(A_{1} \times\{1\}\right)$ and $B=\left(B_{1} \times\{0\}\right) \cup\left(B_{1} \times\{1\}\right)$ and insert an edge between $(a, i) \in A$ and $(b, j) \in B$ whenever there is an edge between $a$ and $b$ in $H$. We let $A^{\prime}=A_{1} \times\{0\} \subseteq A$ and $B^{\prime}=B_{1} \times\{0\} \subseteq B$. Note that because $H$ is the union of 20 matchings, $G$ has maximum degree at most 40 , even after duplicating the vertices.

To complete the proof, we need to show that for any $Z \subseteq A^{\prime} \cup B^{\prime}$ with $\left|Z \cap A^{\prime}\right|=\left|Z \cap B^{\prime}\right|$, the graph $G[V(G) \backslash Z]$ contains a perfect matching. Let $m:=\left|Z \cap A^{\prime}\right|=\left|Z \cap B^{\prime}\right|$ and set $A_{Z}:=A \backslash Z$ and $B_{Z}:=B \backslash Z$. We show that $G[V(G) \backslash Z]$ contains a perfect matching by verifying Hall's condition, i.e., by showing that for every set $X \subseteq A_{Z}$, we have $\left|N_{G}\left(X, B_{Z}\right)\right| \geqslant|X|$.

Assume first $|X| \leqslant n / 2$. Let $X^{\prime}$ be the larger of the sets $A^{\prime} \cap X$ and $\left(A \backslash A^{\prime}\right) \cap X$. Then $|X| / 2 \leqslant\left|X^{\prime}\right| \leqslant n / 2$. By $(i)$ we have $\left|N_{G}\left(X, B_{Z}\right)\right| \geqslant\left|N_{G}\left(X^{\prime}, B_{Z}\right)\right| \geqslant\left|N_{H}\left(X^{\prime}\right)\right| \geqslant 2\left|X^{\prime}\right| \geqslant|X|$.

Suppose next $n / 2<|X| \leqslant 3 n / 2-m$. Let $X^{\prime}$ be the larger of the sets $A^{\prime} \cap X$ and $\left(A \backslash A^{\prime}\right) \cap X$, and note $\left|X^{\prime}\right| \geqslant n / 4$. By definition, there are no edges between $X$ and $Y:=B_{Z} \backslash N_{G}\left(X, B_{Z}\right)$. If we assume $\left|N_{G}\left(X, B_{Z}\right)\right| \leqslant|X|$, then we have $|Y| \geqslant(2 n-m)-(3 n / 2-m) \geqslant n / 2$. Let $Y^{\prime}$ be the larger of the sets $B^{\prime} \cap Y$ and $\left(B \backslash B^{\prime}\right) \cap Y$. The fact that there are no edges between $X^{\prime}$ and $Y^{\prime}$ then contradicts $($ iii).

Finally, suppose $3 n / 2-m<|X| \leqslant 2 n-m$. Assume towards contradiction that $N_{G}\left(X, B_{Z}\right)$ is contained in a set $Q \subseteq B_{Z}$ of size $|X|-1$ and let $Y:=B_{Z} \backslash Q$. Note that $|Y|=2 n-m-(|X|-1) \leqslant$ 
$n / 2$. Let $Y^{\prime}$ be the larger of the sets $B^{\prime} \cap Y$ and $\left(B \backslash B^{\prime}\right) \cap Y$ and thus $\left|Y^{\prime}\right| \geqslant|Y| / 2$. However, all neighbours of $Y^{\prime}$ are contained in the set $A_{Z} \backslash X$ of size $2 n-m-|X|=|Y|-1$. This contradicts $(i i)$.

We are ready to give a proof of the Absorber Lemma. We first restate it for convenience.

Lemma 3.7 (Absorbing Lemma). For every $\gamma \in(0,1)$, there exists a positive $C(\gamma)$ such that the following holds for all sufficiently large $n$. Let $p \in(0,1)$ and $\beta>0$, let $G=(A, B, E)$ be a bipartite $(p, \beta)$-sparse graph on $n$ vertices, and let $U, W \subseteq V(G)$ be disjoint subsets such that:

(i) $G[W]$ is a $\gamma p$-expander,

(ii) $|W| \geqslant C \cdot \max \left\{\log n / p, \beta \sqrt{\log n} / p,|U| \log ^{2} n\right\}$, and

(iii) $|U| \geqslant 2$ and every vertex $u \in U$ satisfies $\operatorname{deg}(u, W) \geqslant \gamma|W| p$.

Then $G[U \cup W]$ contains a $(U \cap A, U \cap B)$-absorber with one endpoint in $W \cap A$ and another in $W \cap B$.

Proof of Lemma 3.7. Let $\gamma_{1}=\gamma_{3.5}(\gamma)$ and $C=4 \cdot \max \left\{C_{3.5}(\gamma), C_{3.4}\left(\gamma_{1}, 80\right)\right\}$. Let us write $W_{A}:=W \cap A$ and $W_{B}:=W \cap B$. We first show that both of these sets have size at least $\gamma|W| / 4$. Indeed, if we assume that, say, $\left|W_{A}\right| \leqslant \gamma|W| / 4$, we have

$$
\gamma|W|^{2} p / 3 \leqslant e_{G}\left(W_{A}, W_{B}\right) \leqslant p\left|W_{A}\right|\left|W_{B}\right|+\beta \sqrt{\left|W_{A}\right|\left|W_{B}\right|} \leqslant \gamma p|W|^{2} / 4+\beta|W|<\gamma p|W|^{2} / 3,
$$

where in the first inequality we use the fact that $G$ is a $\gamma p$-expander, in the second that it is a $(p, \beta)$-sparse graph, and in the last that $|W| \geqslant C \beta \sqrt{\log n} / p$. Thus, by an analogous argument for $\left|W_{B}\right|$, we conclude

$$
\left|W_{A}\right| \geqslant \gamma|W| / 4 \quad \text { and } \quad\left|W_{B}\right| \geqslant \gamma|W| / 4 .
$$

Let now $a$ and $b$ be two arbitrary vertices such that $a \in W_{A}$ and $b \in W_{B}$. These vertices are going to be the endpoints of our absorber. We start by making some preparations.

Let $m:=\max \{|U \cap A|,|U \cap B|\}$ and let $W_{A}^{\prime} \subseteq W_{A} \backslash\{a\}$ and $W_{B}^{\prime} \subseteq W_{B} \backslash\{b\}$ be subsets with $\left|W_{A}^{\prime}\right|=2 m-|U \cap A|$ and $\left|W_{B}^{\prime}\right|=2 m-|U \cap B|$ chosen uniformly at random among all subsets of this size. Note that it is possible to choose subsets of this size because of (13) and the assumption $|U| \leqslant|W| /\left(C \log ^{2} n\right)$. In the following, we write $U_{A}:=W_{A}^{\prime} \cup(U \cap A)$ and $U_{B}:=W_{B}^{\prime} \cup(U \cap B)$; both of these sets have size $2 m$.

Furthermore, let $W_{1} \cup W_{2} \cup W_{3}=W \backslash\left(W_{A}^{\prime} \cup W_{B}^{\prime} \cup\{a, b\}\right)$ be an equipartition chosen uniformly at random. Since $\left|W_{A}^{\prime}\right|,\left|W_{B}^{\prime}\right| \leqslant 2|U| \leqslant|W| / \log ^{2} n$, we have $\left|W_{i}\right| \geqslant|W| / 4$. Therefore, by our choice of $C$, the assumptions of the lemma together with the Inheritance Lemma (Lemma 3.5), Chernoff's inequality, and the union bound, ensure that w.h.p. the following three properties are satisfied for all $i \in[3]$ :

(i) $G\left[W_{i}\right]$ is a $\gamma_{1} p$-expander,

(ii) $\left|W_{i}\right| \geqslant(C / 4) \cdot \beta \sqrt{\log n} / p$, and

(iii) for every vertex $u \in U \cup W$, we have $\operatorname{deg}\left(u, W_{i}\right) \geqslant \gamma_{1}\left|W_{i}\right| p$.

In the following we assume that these properties hold deterministically for all $W_{i}$.

Let $G_{T}=\left(A_{T}, B_{T}, E_{T}\right)$ with $\left|A_{T}\right|=\left|B_{T}\right|=2 m$ and $\Delta\left(G_{T}\right) \leqslant 40$ be a graph given by Lemma 6.1. Furthermore, let $A_{T}^{\prime} \subseteq A_{T}$ and $B_{T}^{\prime} \subseteq B_{T}$ with $\left|A_{T}^{\prime}\right|=\left|B_{T}^{\prime}\right|=m$ be subsets given by the former lemma with the property that for every $Z \subseteq A_{T}^{\prime} \cup B_{T}^{\prime}$ with $\left|Z \cap A_{T}^{\prime}\right|=\left|Z \cap B_{T}^{\prime}\right|$, the graph $G_{T}\left[V\left(G_{T}\right) \backslash Z\right]$ contains a perfect matching. Lastly, let $f: V\left(G_{T}\right) \rightarrow U_{A} \cup U_{B}$ be a bijection mapping the vertices of $A_{T}$ onto $U_{A}$ and $B_{T}$ onto $U_{B}$ and such that $U \cap A \subseteq f\left(A_{T}^{\prime}\right)$ and $U \cap B \subseteq f\left(B_{T}^{\prime}\right)$.

The construction of the absorber now proceeds by three independent applications of the Connecting Lemma. Set $\ell=\left\lceil 30 \log n /\left(\gamma_{1} \log \log n\right)\right\rceil$. Firstly, we apply it with $\gamma_{1}($ as $\gamma), U_{A} \cup U_{B}$ (as $U$ ), $W_{1}$ (as $W$ ), and with the multigraph $M$ with the vertex set $U_{A} \cup U_{B}$ and the edge set 
defined as follows: for every edge $\{x, y\} \in E\left(G_{T}\right)$, add a double edge $\{f(x), f(y)\}$ to $M$. Since $\Delta\left(G_{T}\right) \leqslant 40$, it follows that $\Delta(M) \leqslant 80$. Moreover, the assumption $|U| \leqslant|W| /\left(C \log ^{2} n\right) \leqslant$ $4\left|W_{1}\right| /\left(C \log ^{2} n\right)$ shows that the assumption $e(M) \leqslant\left|W_{1}\right| /(C \ell)$ is satisfied. Finally, $(i)-(i i i)$ show that assumptions Lemma $3.4($ i $)$-(iii) are satisfied. Therefore, we obtain for every edge $\{x, y\} \in E\left(G_{T}\right)$ two $f(x) f(y)$-paths of length at most $\ell$ such that all paths are internally vertexdisjoint and use only vertices from $W_{1}$. For a given edge $\{x, y\} \in E\left(G_{T}\right)$, we denote these two $f(x) f(y)$-paths by $P_{x y}$ and $Q_{x y}$ and let

$$
P_{x y}=f(x), a_{p}^{1}, b_{p}^{1}, a_{p}^{2}, \ldots, a_{p}^{\ell_{p}}, b_{p}^{\ell_{p}}, f(y) \quad \text { and } \quad Q_{x y}=f(x), a_{q}^{1}, b_{q}^{1}, a_{q}^{2}, \ldots, a_{q}^{\ell_{q}}, b_{q}^{\ell_{q}}, f(y),
$$

where $\ell_{p}=\left(\left|P_{x y}\right|-2\right) / 2, \ell_{q}=\left(\left|Q_{x y}\right|-2\right) / 2, \ell_{q} \geqslant \ell_{p}$ (w.l.o.g.), and $a_{p}^{i}, a_{q}^{j} \in A$ and $b_{p}^{i}, b_{q}^{j} \in B$. Note that since $f(x)$ and $f(y)$ lie in different colour classes of $G$, both paths necessarily have odd length.

Given a collection of paths $\mathcal{P}=\left\{P_{x y}, Q_{x y}:\{x, y\} \in E\left(G_{T}\right)\right\}$ as above, let

$$
U_{\mathcal{P}}:=\bigcup_{\{x, y\} \in E_{T}} V\left(P_{x y}\right) \cup V\left(Q_{x y}\right) \backslash\{f(x), f(y)\} .
$$

Next, we apply the Connecting Lemma with $\gamma_{1}$ (as $\left.\gamma\right), U_{\mathcal{P}}$ (as $\left.U\right), W_{2}$ (as $W$ ), and a multigraph $M$ defined as follows: the vertex set of $M$ is just $U_{\mathcal{P}}$ and the edge set is the union of

$$
\begin{aligned}
E\left(M_{x y}\right):=\{ & \left\{b_{p}^{i}, a_{q}^{i}\right\}_{1 \leqslant i \leqslant \ell_{p}},\left\{a_{p}^{i+1}, b_{q}^{i}\right\}_{1 \leqslant i \leqslant \ell_{p}-1}, \\
& \left.\left\{b_{q}^{\ell_{p}+i-1}, b_{q}^{\ell_{q}-i+1}\right\}_{1 \leqslant i \leqslant\left[\left(\ell_{q}-\ell_{p}\right) / 2\right]},\left\{a_{q}^{\ell_{p}+i}, a_{q}^{\ell_{q}-i+1}\right\}_{1 \leqslant i \leqslant\left[\left(\ell_{q}-\ell_{p}\right) / 2\right]}\right\},
\end{aligned}
$$

for all $\{x, y\} \in E\left(G_{T}\right)$. The edge set is much easier to 'define' visually - it is given by the dashed lines in Figure 1 for every $\{x, y\} \in E\left(G_{T}\right)$.

It is easy to verify that the assumptions of the Connecting Lemma are all satisfied-perhaps the least evident being that $e(M) \leqslant\left|W_{2}\right| /(C \ell)$, which holds because $M$ has at most $e\left(G_{T}\right) \cdot 4 \ell \leqslant$ $320 m \ell \leqslant 320|U| \ell$ edges and $|U| \leqslant|W| /\left(C \log ^{2} n\right) \leqslant\left|W_{2}\right| /\left(320 C \ell^{2}\right)$. Therefore, we obtain an $\left(M, W_{2}, \ell\right)$-matching, that is replace the dashed edges in Figure 1, for all $\{x, y\} \in E\left(G_{T}\right)$, by internally vertex-disjoint paths in $G$ whose internal vertices all belong to $W_{2}$.

Lastly, for every $\{x, y\} \in E\left(G_{T}\right)$ denote by $u_{x y}$ and $v_{x y}$ the vertices $a_{p}^{1}$ and $b_{q}^{\left(\ell_{p}+\ell_{q}\right) / 2}$ (assuming $\ell_{q}$ is even, otherwise this is $\left.a_{q}^{\left(\ell_{p}+\ell_{q}+1\right) / 2}\right)$, respectively (the ones as in Figure 1) and let us fix an arbitrary ordering $\left\{x_{1}, y_{1}\right\}, \ldots,\left\{x_{m_{T}}, y_{m_{T}}\right\}$ of the edges of $G_{T}$, where $m_{T}:=e\left(G_{T}\right)$. We now apply the Connecting Lemma for the third and last time with $V(M):=\left\{u_{x_{i} y_{i}}, v_{x_{i} y_{i}}\right\}_{i \in\left[m_{T}\right]} \cup\{a, b\}$ (as $U$ ), $W_{3}$ (as $W$ ), and the edge set of $M$

$$
E(M):=\left\{\left\{a, u_{x_{1} y_{1}}\right\},\left\{v_{x_{i} y_{i}}, u_{x_{i+1} y_{i+1}}\right\}_{i \in\left[m_{T}-1\right]},\left\{v_{x_{m_{T}} y_{m_{T}}}, b\right\}\right\} .
$$

Similarly as above, one easily checks that all the assumptions of the Connecting Lemma are satisfied and hence we obtain an $\left(M, W_{3}, \ell\right)$-matching which connects all the two-vertex absorbers into one large absorber $H$. For a more natural, visual representation we depict the obtained structure on Figure 2.

It remains to show that the graph constructed in this way is a $(U \cap A, U \cap B)$-absorber with endpoints $a$ and $b$. For this, suppose that $A^{\prime} \subseteq U \cap A$ and $B^{\prime} \subseteq U \cap B$ are subsets such that $\left|A^{\prime}\right|=\left|B^{\prime}\right|$. Then we let $Z:=f^{-1}\left(A^{\prime} \cup B^{\prime}\right)$ and note that $Z$ is a subset of $A_{T}^{\prime} \cup B_{T}^{\prime}$ that intersects each set $A_{T}^{\prime}$ and $B_{T}^{\prime}$ in the same number of vertices. Hence, by the defining property of $G_{T}$, the graph $G_{T}\left[V\left(G_{T}\right) \backslash Z\right]$ contains a perfect matching $M$. We can then find an ab-path using all vertices except those in $A^{\prime} \cup B^{\prime}$, as follows: for each edge $\{x, y\}$ in the given perfect matching take a $u_{x y} v_{x y}$-path which includes all vertices of $H_{x y}$; for all other edges take a $u_{x y} v_{x y}$-path which includes all vertices of $H_{x y}$ except for $f(x)$ and $f(y)$. Since the edges in $M$ form a perfect matching, it is clear that this is indeed a path (that is, no vertex is used twice) and that this path visits each vertex of the absorber except those contained in the set $A^{\prime} \cup B^{\prime}$. 


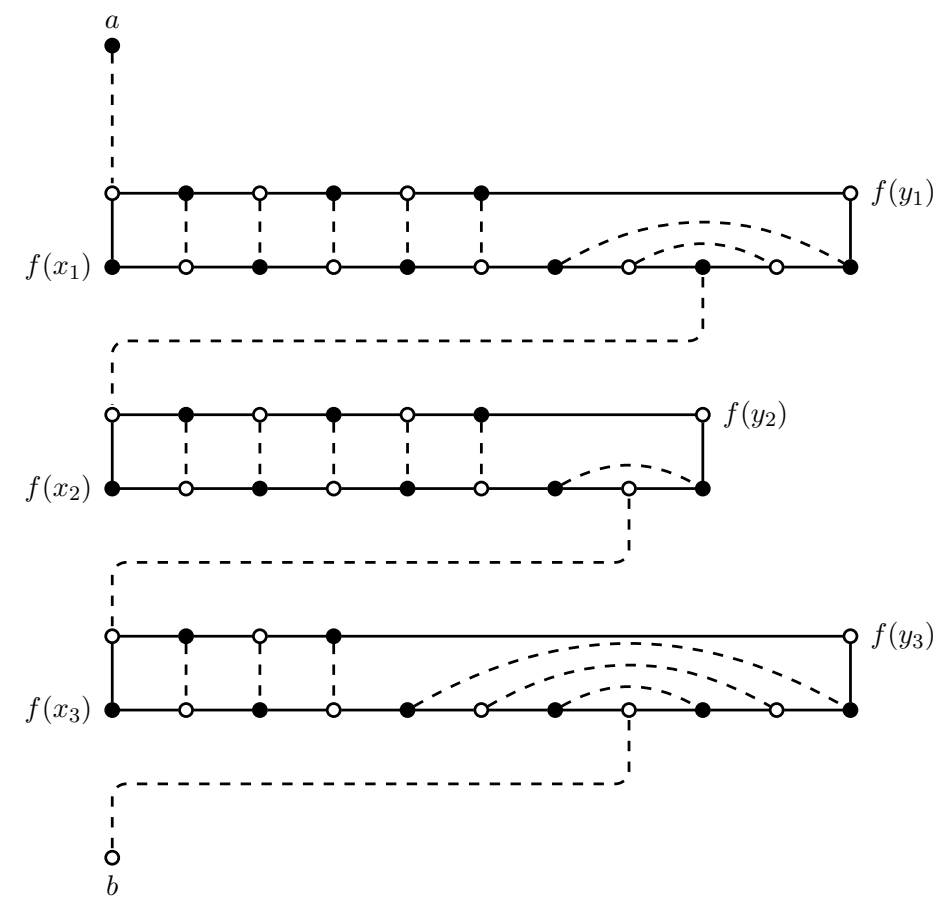

Figure 2. The absorber $H$. The vertices $f\left(x_{1}\right), f\left(x_{2}\right), f\left(x_{3}\right)$ and respectively $f\left(y_{1}\right), f\left(y_{2}\right), f\left(y_{3}\right)$ are not necessarily distinct (they would be distinct only if $G_{T}$ were itself a perfect matching); however, all other vertices are actually distinct.

\section{The Partitioning Lemma}

In this section we give a proof of the Partitioning Lemma which allows us to partition every $(p, o(n p))$-sparse graph satisfying a certain minimum degree condition into linear-sized expanders. The proof uses the following notions of good and perfect partitions.

Definition $7.1((c, \alpha)$-good, $(c, \alpha, \gamma)$-perfect). Let $G$ be a graph on $n$ vertices. We say that a partition $V(G)=V_{0} \cup \cdots \cup V_{\ell}$ of the vertex set of $G$ is $(c, \alpha)$-good if

(L1) $\left|V_{0}\right| \leqslant \alpha n$, and

(L2) $\delta\left(G\left[V_{i}\right]\right) \geqslant\left(c+\alpha / 2^{\ell}\right) n p$ for every $i \in[\ell]$.

We say that the partition is $(c, \alpha, \gamma)$-perfect if it additionally satisfies

(L3) $G\left[V_{i}\right]$ is a $\gamma p$-expander for every $i \in[\ell]$.

A first step towards proving Lemma 3.1 is proving the following auxiliary lemma.

Lemma 7.2. For all $c, \alpha \in(0,1)$, there exist positive $\gamma(\alpha, c)$ and $\eta(\alpha, c)$ such that the following holds for all sufficiently large $n$. Let $p \in(0,1)$ and $\beta \leqslant \eta n p$ and let $G$ be $a(p, \beta)$-sparse graph with $n$ vertices and minimum degree at least $(c+\alpha) n p$. Then for some integer $1 \leqslant \ell<1 / c$, there exists a $(c, \alpha, \gamma)$-perfect partition $V(G)=V_{0} \cup V_{1} \cup \cdots \cup V_{\ell}$ of the vertex set of $G$.

Proof. Let $G=(V, E)$ and let $\gamma=\alpha^{2} / 2^{2 / c+1}$. Suppose that $\eta=\eta(c, \alpha)$ is sufficiently small for the rest of the argument to go through. For every $\ell \geqslant 1$, set

$$
c_{\ell}=c+\alpha / 2^{\ell-1} \geqslant c, \quad \alpha_{\ell}=\alpha\left(2^{-1}+2^{-2}+\cdots+2^{-\ell}\right) \leqslant \alpha, \quad \text { and } \quad \gamma_{\ell}=\alpha^{2} / 2^{2 \ell+1} .
$$

Note that the partition $V=V_{0} \cup V_{1}$ where $V_{0}=\varnothing$ is trivially $\left(c_{1}, \alpha_{1}\right)$-good. In the following we argue that if for some $\ell<c^{-1}$ a partition $V=V_{0} \cup \cdots \cup V_{\ell}$ is $\left(c_{\ell}, \alpha_{\ell}\right)$-good, but not $\left(c_{\ell}, \alpha_{\ell}, \gamma_{\ell}\right)$ perfect, then there exists a partition $V=W_{0} \cup \cdots \cup W_{\ell+1}$ that is $\left(c_{\ell+1}, \alpha_{\ell+1}\right)$-good. This is sufficient to complete the proof, which can be seen as follows. By repeatedly applying the statement above, we either obtain a $\left(c_{\ell}, \alpha_{\ell}, \gamma_{\ell}\right)$-perfect partition $V=V_{0} \cup \cdots \cup V_{\ell}$ for some 
$1 \leqslant \ell<c^{-1}$ or we obtain a $\left(c_{\ell}, \alpha_{\ell}\right)$-good partition $V=V_{0} \cup \cdots \cup V_{\ell}$ for $\ell=\left\lceil c^{-1}\right\rceil$. In the former case, we are done because $c_{\ell}=c+\alpha / 2^{\ell-1}, \alpha_{\ell} \leqslant \alpha$, and $\gamma_{\ell} \geqslant \gamma$ for $\ell<c^{-1}$. The latter case results in a contradiction, as we now verify. By averaging, there is some $i \in[\ell]$ such that $\left|V_{i}\right| \leqslant n / \ell$. Since $\ell \geqslant c^{-1}$, we then have $\left|V_{i}\right| \leqslant c n$. Furthermore, (L2) states that $\delta\left(G\left[V_{i}\right]\right) \geqslant\left(c+\alpha / 2^{\ell-1}\right) n p \geqslant\left(c+\alpha / 2^{1 / c}\right) n p$, which in particular implies

$$
2 e\left(V_{i}\right) \geqslant\left|V_{i}\right|\left(c+\alpha / 2^{1 / c}\right) n p .
$$

On the other hand, since $G$ is $(p, \beta)$-sparse, we have $2 e\left(V_{i}\right)=e\left(V_{i}, V_{i}\right) \leqslant p\left|V_{i}\right|^{2}+\beta\left|V_{i}\right|$, which combines with the above to yield

$$
\left(c+\alpha / 2^{1 / c}\right) n p \leqslant p\left|V_{i}\right|+\beta .
$$

Since $\left|V_{i}\right| \leqslant c n$ and $\beta \leqslant \eta n p$, this is a contradiction if $\eta<\alpha / 2^{1 / c}$.

We now prove the statement mentioned above. Assume that the partition $V=V_{0} \cup \cdots \cup V_{\ell}$ is $\left(c_{\ell}, \alpha_{\ell}\right)$-good but not $\left(c_{\ell}, \alpha_{\ell}, \gamma_{\ell}\right)$-perfect, for some $\ell<c^{-1}$. Then there is some $i \in[\ell]$ and a partition $V_{i}=X \cup Y$ such that

$$
e(X, Y)<\gamma_{\ell}|X||Y| p .
$$

We further define partitions $X=V_{X} \cup W_{X}$ and $Y=V_{Y} \cup W_{Y}$ as follows. Set

$$
W_{X}^{0}:=\left\{v \in X: \operatorname{deg}(v, Y) \geqslant \alpha n p / 2^{\ell}\right\},
$$

and, for every $j \geqslant 1$, set

$$
W_{X}^{j}:= \begin{cases}W_{X}^{j-1} \cup\{v\}, & \text { if there exists } v \in X \backslash W_{X}^{j-1} \text { with } \operatorname{deg}\left(v, W_{X}^{j-1}\right) \geqslant \alpha n p / 2^{\ell}, \\ W_{X}^{j-1}, & \text { otherwise. }\end{cases}
$$

Finally, define $W_{X}:=\bigcup_{j \geqslant 0} W_{X}^{j}$ and $V_{X}:=X \backslash W_{X}$. The partition $Y=V_{Y} \cup W_{Y}$ is defined analogously (with $X$ replaced by $Y$ ).

We now define the partition $V=W_{0} \cup \cdots \cup W_{\ell+1}$ by setting $W_{0}=V_{0} \cup W_{X} \cup W_{Y}$ and $\left(W_{1}, \ldots, W_{\ell+1}\right)=\left(V_{1}, \ldots, V_{i-1}, V_{X}, V_{Y}, V_{i+1}, \ldots, V_{\ell}\right)$, i.e., we obtain the new partition of $V$ by replacing the part $V_{i}$ by the two parts $V_{X}$ and $V_{Y}$ and adding $W_{X} \cup W_{Y}$ to $V_{0}$. It remains to check that this partition is $\left(c_{\ell+1}, \alpha_{\ell+1}\right)$-good.

Showing (L1) essentially boils down to proving that $W_{X}$ and $W_{Y}$ are not too large. As a first step, it follows from (14) and (15) that

$$
\left|W_{X}^{0}\right| \cdot \alpha n p / 2^{\ell} \leqslant e(X, Y)<\gamma_{\ell}|X||Y| p \leqslant \gamma_{\ell}\left|V_{i}\right|^{2} p / 4,
$$

and thus $\left|W_{X}^{0}\right| \leqslant 2^{\ell-2} \gamma_{\ell}\left|V_{i}\right| / \alpha \leqslant 2^{\ell-2} \gamma_{\ell} n / \alpha$. Assume towards a contradiction that $\left|W_{X}\right|>$ $\alpha n / 2^{\ell+2}$. Then there must exist some $j \geqslant 0$ such that $\left|W_{X}^{j}\right|=\left\lceil\alpha n / 2^{\ell+2}\right\rceil$. We remark that by the choice $\gamma_{\ell}=\alpha^{2} / 2^{2 \ell+1}$, we have $\left|W_{X}^{0}\right| \leqslant 2^{\ell-2} \gamma_{\ell} n / a=\alpha n / 2^{\ell+3} \leqslant\left|W_{X}^{j}\right| / 2$. From the definition of $W_{X}^{j}$, we moreover see that every vertex in $W_{X}^{j} \backslash W_{X}^{0}$ adds at least $\alpha n p / 2^{\ell}$ edges to $e\left(W_{X}^{j}\right)$. Therefore, we have

$$
e\left(W_{X}^{j}\right) \geqslant \frac{\alpha}{2^{\ell}} n p \cdot\left|W_{X}^{j} \backslash W_{X}^{0}\right| \geqslant \frac{\alpha}{2^{\ell+1}} n p \cdot\left|W_{X}^{j}\right| .
$$

On the other hand, since $G$ is $(p, \beta)$-sparse,

$$
e\left(W_{X}^{j}\right) \leqslant\left|W_{X}^{j}\right|^{2} p / 2+\beta\left|W_{X}^{j}\right|=\left\lceil\frac{\alpha n}{2^{\ell+2}}\right\rceil\left(\left|W_{X}^{j}\right| p / 2+\beta\right) .
$$

As $\beta \leqslant \eta n p \leqslant \eta 2^{\ell+2}\left|W_{X}^{j}\right| p / \alpha$, we see that for small enough $\eta$, equations (17) and (18) result in a contradiction. It follows that $\left|W_{X}\right| \leqslant \alpha n / 2^{\ell+2}$ and one can show analogously that $\left|W_{Y}\right| \leqslant$ $\alpha n / 2^{\ell+2}$. In conclusion,

$$
\left|W_{0}\right|=\left|V_{0}\right|+\left|W_{X}\right|+\left|W_{Y}\right| \leqslant\left(\alpha_{\ell}+\alpha / 2^{\ell+1}\right) n=\alpha_{\ell+1} n,
$$

completing the proof of (L1). 
Lastly, we prove (L2). Since $V=V_{0} \cup \cdots \cup V_{\ell}$ is $\left(c_{\ell}, \alpha_{\ell}\right)$-good, we have $\delta\left(G\left[V_{i}\right]\right) \geqslant c_{\ell} n p=$ $\left(c+\alpha / 2^{\ell-1}\right) n p$. Observe that (15) and (16) imply that $V_{X}$ contains only vertices with fewer than $\alpha n p / 2^{\ell}$ neighbours in $W_{X} \cup Y=V_{i} \backslash V_{X}$. Therefore,

$$
\delta\left(G\left[V_{X}\right]\right) \geqslant\left(c+\alpha / 2^{\ell-1}\right) n p-\alpha n p / 2^{\ell}=\left(c+\alpha / 2^{\ell}\right) n p=c_{\ell+1} n p .
$$

Similarly, we prove $\delta\left(G\left[V_{Y}\right]\right) \geqslant c_{\ell+1} n p$, thus establishing (L2).

We can now prove Lemma 3.1 which we restate for convenience.

Lemma 3.1 (Partitioning Lemma). For all $c, \alpha, \xi \in(0,1)$, there exist positive $\gamma(\alpha, \xi, c)$ and $\eta(\alpha, \xi, c)$ such that the following holds for all sufficiently large $n$. Let $p \in(0,1)$ and $\beta \leqslant \eta n p$, and let $G$ be a $(p, \beta)$-sparse graph on $n$ vertices with minimum degree at least $(c+\alpha) n p$. Then there exists a partition $V(G)=V_{1} \cup \cdots \cup V_{\ell}$ into $1 \leqslant \ell<1 / c$ parts such that, for every $i \in[\ell]$,

(i) $G\left[V_{i}\right]$ is a $\gamma p$-expander,

(ii) $\delta\left(G\left[V_{i}\right]\right) \geqslant c^{2} n p$,

(iii) $\delta_{\xi}\left(G\left[V_{i}\right]\right) \geqslant(c+\alpha-\xi) n p$.

Proof. We may assume without loss of generality that $\xi<\alpha$. Let $c^{\prime}=c+\alpha-\xi, \alpha^{\prime}=c^{2} \xi / 4$, and choose $\eta$ to be small enough so that the following arguments hold. Then $G$ has minimum degree at least $\left(c^{\prime}+\alpha^{\prime}\right) n p$, and so we can apply Lemma 7.2 to $G$ to obtain, for some $\gamma^{\prime}>0$ and some integer $1 \leqslant \ell<c^{-1}$, a $\left(c^{\prime}, \alpha^{\prime}, \gamma^{\prime}\right)$-perfect partition $V(G)=V_{0}^{\prime} \cup V_{1}^{\prime} \cup \cdots \cup V_{\ell}^{\prime}$. In the following, we distribute the vertices in $V_{0}^{\prime}$ over the other sets to obtain a partition $V(G)=V_{1} \cup \cdots \cup V_{\ell}$ as in the statement of the lemma (in particular, we aim to have $V_{i}^{\prime} \subseteq V_{i}$ for every $i \in[\ell]$ ).

Let $m=\left|V_{0}^{\prime}\right|$ and note that by (L1), we have $m \leqslant \alpha^{\prime} n$. It then follows from the fact that $G$ has minimum degree at least $\left(c^{\prime}+\alpha^{\prime}\right) n p \geqslant\left|V_{0}^{\prime}\right| p+c^{\prime} n p$ and Lemma 2.1 that there exists an ordering $w_{1}, \ldots, w_{m}$ of the vertices of $V_{0}^{\prime}$ such that for every $j \in[m]$, we have

$$
\operatorname{deg}\left(w_{j}, V \backslash\left\{w_{j}, \ldots, w_{m}\right\}\right) \geqslant c^{\prime} n p-\beta \geqslant c n p,
$$

where the last inequality holds by choosing $\eta$ to be small enough. We process the vertices $w_{1}, \ldots, w_{m}$ in this order, defining $\ell$ chains of subsets

$$
\varnothing=W_{i}^{0} \subseteq W_{i}^{1} \subseteq \cdots \subseteq W_{i}^{m} \subseteq V_{0}^{\prime} \quad \text { for } i \in[\ell]
$$

along the way. For this, we set $W_{i}^{0}=\varnothing$ for every $i \in[\ell]$, and for every vertex $w_{j}$, we do the following:

(1) choose an arbitrary $i^{\star} \in[\ell]$ satisfying $\operatorname{deg}\left(w_{j}, V_{i^{\star}}^{\prime} \cup W_{i^{\star}}^{j-1}\right) \geqslant c n p / \ell$,

(2) set $W_{i^{\star}}^{j}:=W_{i^{\star}}^{j-1} \cup\left\{w_{j}\right\}$ and $W_{i}^{j}:=W_{i}^{j-1}$ for all $i \neq i^{\star}$.

Observe that by (19) there always exists at least one $i^{\star} \in[\ell]$ as in (1). Lastly, we define $V_{i}:=W_{i}^{m} \cup V_{i}^{\prime}$ for every $i \in[\ell]$.

It is easy to see that $V_{1}, \ldots, V_{\ell}$ contain all vertices of $V_{0}^{\prime}$ and that we have $\delta\left(G\left[V_{i}\right]\right) \geqslant c n p / \ell \geqslant$ $c^{2} n p$ for every $i \in[\ell]$. Moreover, by (L2), all but at most $\left|V_{0}^{\prime}\right| \leqslant \alpha^{\prime} n$ vertices $v$ in each set $V_{i}$ satisfy $\operatorname{deg}\left(v, V_{i}\right) \geqslant c^{\prime} n p=(c+\alpha-\xi) n p$. Since $G$ is $(p, \beta)$-sparse and $\delta\left(G\left[V_{i}\right]\right) \geqslant c^{2} n p$, one easily derives $\left|V_{i}\right| \geqslant c^{2} n / 2$. Thus, by our choice of constants $\alpha^{\prime} n \leqslant \xi\left|V_{i}\right|$ and so $\delta_{\xi}\left(G\left[V_{i}\right]\right) \geqslant(c+\alpha-\xi) n p$. We finish the proof by showing that each graph $G\left[V_{i}\right]$ is a $\gamma p$-expander, with $\gamma=\min \left\{c^{2} / 4, \gamma^{\prime} / 4\right\}$.

For this, fix some partition $V_{i}=X \cup Y$ into non-empty sets where, without loss of generality, we assume $|X| \leqslant|Y|$. If $|X| \leqslant c^{2} n / 2$, then it follows from $\delta\left(G\left[V_{i}\right]\right) \geqslant c^{2} n p \geqslant|X| p+c^{2} n p / 2$ and Lemma 2.1 that $e(X, Y) \geqslant c^{2}|X| n p / 2-\beta|X| \geqslant \gamma|X||Y| p$, for $\eta$ small enough. On the other hand, if $|X|,|Y| \geqslant c^{2} n / 2$, then we use the fact that $G\left[V_{i}^{\prime}\right]$ is a $\gamma^{\prime} p$-expander to get

$$
e(X, Y) \geqslant e\left(X \cap V_{i}^{\prime}, Y \cap V_{i}^{\prime}\right) \geqslant \gamma^{\prime}\left|X \cap V_{i}^{\prime}\right|\left|Y \cap V_{i}^{\prime}\right| p .
$$


The assumption on the sizes of $X$ and $Y$ implies $\left|X \cap V_{i}^{\prime}\right|=\left|X \backslash V_{0}^{\prime}\right| \geqslant|X|-\alpha^{\prime} n \geqslant|X| / 2$ and similarly $\left|Y \cap V_{i}^{\prime}\right| \geqslant|Y| / 2$. This gives

$$
e(X, Y) \geqslant \gamma^{\prime}|X||Y| p / 4 \geqslant \gamma|X||Y| p
$$

as required.

\section{EMBEDding AND BOOsting}

In this section we give the proof of the Embedding Lemma (Lemma 3.8). The proof relies on the following approximate version covering almost all the vertices of $G$.

Lemma 8.1. For every integer $k \geqslant 2$ and all $\alpha, \mu>0$, there exists a positive $\eta(\alpha, k)$ such that the following holds for all sufficiently large $n$. Let $p \in(0,1)$ and $\beta \leqslant \eta n p$ and let $G$ be a $(p, \beta)$-sparse graph on $n$ vertices such that

$$
\delta(G) \geqslant 2 \alpha n p \quad \text { and } \quad \delta_{\alpha / 4}(G) \geqslant(1 / k+\alpha) n p .
$$

Then $G$ contains a collection of $k-1$ cycles covering all but at most $\mu$ n vertices.

Proof sketch. Since the argument is fairly standard nowadays, we only give a rough sketch of the proof. We apply the sparse regularity lemma (Lemma 2.5) to the graph $G$ with a sufficiently small parameter $\varepsilon>0$. Let $t$ be the number of vertices in the reduced graph $R$. Since $G$ is $(p, \beta)$-sparse, straightforward calculations show that $\delta(R) \geqslant \alpha t$ and $\delta_{\alpha / 4+\varepsilon}(R) \geqslant(1 / k+\alpha-2 \varepsilon) t$. Let $U$ be the set containing the at most $(\alpha / 4+\varepsilon) t$ vertices (clusters) $v \in V(R)$ with $\operatorname{deg}_{R}(v)<(1 / k+\alpha-2 \varepsilon) t$. A simple greedy strategy allows us to find a matching $M$ in $R$ that saturates the set $U$; this matching contains at most $2|U| \leqslant(\alpha / 2+2 \varepsilon) t$ vertices. Let $W=V(R) \backslash V(M)$. Since $R[W]$ has minimum degree at least $(1 / k+\alpha-2 \varepsilon) t-2|U| \geqslant t / k$, it can be covered by at most $k-1$ cycles, by Theorem 1.1. Moreover, the minimum degree of $R$ ensures that we can select a different neighbour in $W$ for each of the vertices in $V(M)$.

Using standard machinery, one can now translate each cycle in the covering of $R[W]$, as well as all the matching edges in $M$ that have an endpoint whose selected neighbour lies on that cycle, into a single cycle that covers all but at most $O(\varepsilon n)$ vertices of the graph $G$. The following figure schematically represents one such cycle together with the two edges of $M$ that have an endpoint whose selected neighbour lies on the cycle.

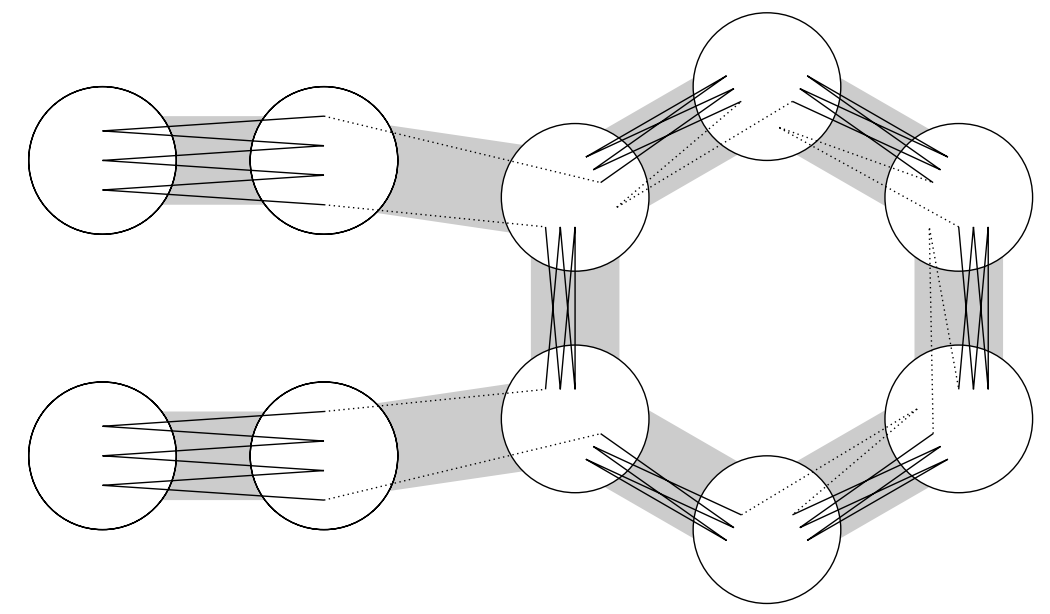

The long black paths - each of which covers all but $O(\varepsilon n / t)$ vertices in both of the clusters it belongs to - are embedded first into the dense regular pairs, using, e.g., the method from the proof of [5, Lemma 2.3]. Finally, using the definition of an $(\varepsilon, p)$-regular pair, the dotted edges can be added by sacrificing at most $O(\varepsilon n / t)$ vertices from each black path. In this way, one obtains a collection of $k-1$ cycles in $G$ covering all but $O(\varepsilon n) \leqslant \mu n$ vertices. 
Utilising a trick of Nenadov and the second author [31], as a consequence of Lemma 8.1 we get Lemma 3.8.

Lemma 3.8 (Embedding Lemma). For every integer $k \geqslant 2$ and every $\alpha>0$, there exists a positive $\eta(\alpha, k)$ such that the following holds for all sufficiently large $n$. Let $p \in(0,1)$ and $\beta \leqslant \eta n p$, and let $G$ be a $(p, \beta)$-sparse graph on $n$ vertices such that

$$
\delta(G) \geqslant 2 \alpha n p \quad \text { and } \quad \delta_{\alpha / 32}(G) \geqslant(1 / k+\alpha) n p .
$$

Then the vertices of $G$ can be covered by $k-1$ path forests $P_{1}, \ldots, P_{k-1}$ that contain at most $\max \{\beta / p, \log n / p\}$ paths each.

Proof. Let $C=C(\alpha, k)>0$ be a sufficiently large constant for the arguments below to go through, $\eta=\eta_{8.1}(\alpha / 4, k)$, and $\mu=\min \{1 / 2, \alpha / 48, \eta / 8,1 /(4 C)\}$.

Take $m$ to be the largest integer such that $n / 2^{m-1}>\max \left\{\left\lfloor\eta^{-1} \beta / p\right\rfloor,\lfloor C \log n / p\rfloor\right\}$ and note that $m \leqslant \log _{2} n$. We first claim that there exists a partition $V(G)=L_{1} \cup \cdots \cup L_{m}$ such that:

(i) $\left|L_{i}\right|=\left\lfloor n / 2^{i}\right\rfloor$ for all $i \in[m-1]$,

(ii) $\operatorname{deg}\left(v, L_{i}\right) \geqslant \alpha\left|L_{i}\right| p$ for all $v \in V(G)$ and $i \in[m]$, and

(iii) for every $i, j \in[m]$, we have $\operatorname{deg}\left(v, L_{i}\right) \geqslant(1 / k+\alpha / 2)\left|L_{i}\right| p$ for all but at most $(\alpha / 24)\left|L_{j}\right|$ vertices $v \in L_{j}$.

Indeed, a partition $V(G)=L_{1} \cup \cdots \cup L_{m}$ chosen uniformly at random among all partitions such that $\left|L_{i}\right|=\left\lfloor n / 2^{i}\right\rfloor$ for all $i \in[m-1]$ has these properties with high probability. We briefly explain how one can conclude this. Observe that

$$
\left|L_{m}\right|=n-\sum_{i=1}^{m-1}\left\lfloor n / 2^{i}\right\rfloor \geqslant n-n \sum_{i=1}^{m-1} 1 / 2^{i}=n / 2^{m-1},
$$

and thus $\left|L_{i}\right| \geqslant C \log n / p$ for all $i \in[m]$. For every fixed $v \in V(G)$ and $i \in[m]$, the random variable $\operatorname{deg}\left(v, L_{i}\right)$ follows a hypergeometric distribution with mean at least $2 \alpha\left|L_{i}\right| p \geqslant 2 \alpha C \log n$, so by Chernoff's inequality, we have $\operatorname{Pr}\left[\operatorname{deg}\left(v, L_{i}\right) \leqslant \alpha\left|L_{i}\right| p\right] \leqslant n^{-2}$. Thus, the union bound shows that (ii) holds with high probability for every $i \in[m]$ and $v \in V(G)$. The statement in (iii) follows similarly.

Fix a partition $V(G)=L_{1} \cup \cdots \cup L_{m}$ satisfying $(i)-(i i i)$. We show by induction that for every $i \in[m]$, the graph $G\left[L_{1} \cup \cdots \cup L_{i}\right]$ contains $k-1$ path forests with at most $i$ paths each, which together cover all but at most $\mu\left|L_{i}\right|$ vertices in $L_{1} \cup \cdots \cup L_{i}$. By maximality of $m$, we have

$$
\left|L_{m}\right| \leqslant n / 2^{m-1}+m \leqslant 2 \max \left\{\eta^{-1} \beta / p, C \log n / p\right\}+\log _{2} n \leqslant 4 \max \left\{\eta^{-1} \beta / p, C \log n / p\right\} .
$$

Hence, for $i=m$, adding at most $\mu\left|L_{m}\right|$ uncovered vertices to one of the path forests used to cover $G\left[L_{1} \cup \cdots \cup L_{m}\right]$ results in a cover of $G$ by $k-1$ path forests, each of which contains at $\operatorname{most}(k-1) m+\mu \cdot 4 \max \left\{\eta^{-1} \beta / p, C \log n / p\right\} \leqslant \max \{\beta / p, \log n / p\}$ paths, for our choice of $\mu$.

For the base case $i=1$, this is a consequence of Lemma 8.1 applied with $\alpha / 2$ (as $\alpha$ ) and $\mu$ to $G\left[L_{1}\right]$. We can indeed do so since $\beta \leqslant \eta\left|L_{1}\right| p$.

Assume now the statement holds for some $1 \leqslant i<m$ and let us show it for $i+1$. Denote by $W$ the vertices not covered by the $k-1$ path forests in $G\left[L_{1} \cup \cdots \cup L_{i}\right]$ and note that $|W| \leqslant \mu\left|L_{i}\right| \leqslant 2 \mu\left|L_{i+1}\right|$. For every $v \in V(G)$ we have

$$
\operatorname{deg}_{G}\left(v, L_{i+1} \cup W\right) \geqslant \operatorname{deg}_{G}\left(v, L_{i+1}\right) \stackrel{(i i)}{\geqslant} \alpha\left|L_{i+1}\right| p \geqslant \alpha \frac{\left|L_{i+1}\right|+|W|}{1+4 \mu} p \geqslant(\alpha / 2)\left|L_{i+1} \cup W\right| p .
$$

Similar calculation shows that every vertex $v \in V(G)$ with $\operatorname{deg}_{G}\left(v, L_{i+1}\right) \geqslant(1 / k+\alpha / 2)\left|L_{i+1}\right| p$ also satisfies $\operatorname{deg}_{G}\left(v, L_{i+1} \cup W\right) \geqslant(1 / k+\alpha / 4)\left|L_{i+1} \cup W\right| p$. Since there were at most $(\alpha / 24)\left|L_{i+1}\right|$ vertices in $L_{i+1}$ violating the previous degree condition by $($ iii $)$, and $|W| \leqslant \mu\left|L_{i+1}\right|$, there are at most $(\alpha / 16)\left|L_{i+1}\right|$ vertices $v \in L_{i+1} \cup W$ with $\operatorname{deg}_{G}\left(v, L_{i+1} \cup W\right)<(1 / k+\alpha / 4)\left|L_{i+1} \cup W\right| p$. Therefore, another application of Lemma 8.1 for $\alpha / 4$ (as $\alpha$ ) to $G\left[L_{i+1} \cup W\right]$ shows that the 
hypothesis holds for $i+1$. Once again, we may apply the lemma as $\beta \leqslant \eta\left|L_{i+1} \cup W\right| p$ by (20).

Acknowledgements. The authors would like to thank the anonymous reviewers for their thorough reading of the paper and valuable comments; in particular, for pointing out a slight gap in a previous version of the paper.

\section{REFERENCES}

[1] P. Allen, J. Böttcher, J. Ehrenmüller, and A. Taraz. The bandwidth theorem in sparse graphs. Advances in Combinatorics, 2020(6):1-60, 2020. 1

[2] J. Balogh, B. Csaba, and W. Samotij. Local resilience of almost spanning trees in random graphs. Random Structures \& Algorithms, 38(1-2):121-139, 2011. 1

[3] J. Balogh, C. Lee, and W. Samotij. Corrádi and Hajnal's theorem for sparse random graphs. Combinatorics, Probability and Computing, 21(1-2):23-55, 2012. 1

[4] J. Balogh, F. Mousset, and J. Skokan. Stability for vertex cycle covers. The Electronic Journal of Combinatorics, 24(3):P3-56, 2017. 1

[5] I. Ben-Eliezer, M. Krivelevich, and B. Sudakov. Long cycles in subgraphs of (pseudo) random directed graphs. Journal of Graph Theory, 70(3):284-296, 2012. 8

[6] J. Böttcher. Large-scale structures in random graphs. Surveys in Combinatorics 2017, 440:87, 2017. 1

[7] J. Böttcher, M. Schacht, and A. Taraz. Proof of the bandwidth conjecture of Bollobás and Komlós. Mathematische Annalen, 343(1):175-205, 2009. 1

[8] P. Châu, L. DeBiasio, and H. A. Kierstead. Pósa's conjecture for graphs of order at least $2 \times 10^{8}$. Random Structures \& Algorithms, 39(4):507-525, 2011. 1

[9] D. Conlon. Combinatorial theorems relative to a random set. In Proceedings of the International Congress of Mathematicians-Seoul 2014. Vol. IV, pages 303-327. Kyung Moon Sa, Seoul, 2014. 1

[10] G. A. Dirac. Some theorems on abstract graphs. Proceedings of the London Mathematical Society, 3(1):69-81, 1952. 1

[11] H. Enomoto, A. Kaneko, and Z. Tuza. $P_{3}$-factors and covering cycles in graphs of minimum degree $n / 3$. In Combinatorics (Eger, 1987), volume 52 of Colloq. Math. Soc. János Bolyai, pages 213-220. North-Holland, Amsterdam, 1988. 1

[12] P. Erdős. Problem 9, Theory of graphs and its applications (M. Fieldler ed.). Czech. Acad. Sci. Publ., Prague, pages $159-159,1964.1$

[13] P. Erdős, A. Gyárfás, and L. Pyber. Vertex coverings by monochromatic cycles and trees. Journal of Combinatorial Theory, Series B, 51(1):90-95, 1991. 1.1

[14] A. Ferber, R. Nenadov, A. Noever, U. Peter, and N. Škorić. Robust Hamiltonicity of random directed graphs. Journal of Combinatorial Theory, Series B, 126:1-23, 2017. 1.1

[15] A. Georgakopoulos, J. Haslegrave, R. Montgomery, and B. Narayanan. Spanning surfaces in 3-graphs. arXiv preprint arXiv:1808.06864, 2018. 1.1

[16] S. Gerke, Y. Kohayakawa, V. Rödl, and A. Steger. Small subsets inherit sparse $\varepsilon$-regularity. Journal of Combinatorial Theory, Series B, 97(1):34-56, 2007. 2.2, 2.6

[17] S. Glock, D. Kühn, R. Montgomery, and D. Osthus. Decompositions into isomorphic rainbow spanning trees. Journal of Combinatorial Theory, Series B, 146:439-484, 2021. 1.1

[18] A. Hajnal and E. Szemerédi. Proof of a conjecture of P. Erdös. Combinatorial theory and its applications, 2:601-623, 1970. 1

[19] P. E. Haxell. A condition for matchability in hypergraphs. Graphs and Combinatorics, 11(3):245-248, 1995. 2.4

[20] J. Komlós, G. N. Sárközy, and E. Szemerédi. Proof of the Seymour conjecture for large graphs. Annals of Combinatorics, 2(1):43-60, 1998. 1

[21] M. Kouider and Z. Lonc. Covering cycles and $k$-term degree sums. Combinatorica, 16(3):407-412, 1996. 1, 1.1

[22] M. Krivelevich. Triangle factors in random graphs. Combinatorics, Probability and Computing, 6(3):337-347, 1997. 1.1

[23] M. Krivelevich and B. Sudakov. Pseudo-random graphs. In More sets, graphs and numbers, pages 199-262. Springer, 2006. 1

[24] M. Kwan. Almost all Steiner triple systems have perfect matchings. Proceedings of the London Mathematical Society, 121(6):1468-1495, 2020. 1.1 
[25] C. Lee and B. Sudakov. Dirac's theorem for random graphs. Random Structures \& Algorithms, 41(3):293-305, 2012. 1

[26] I. Levitt, G. N. Sárközy, and E. Szemerédi. How to avoid using the regularity lemma: Pósa's conjecture revisited. Discrete Mathematics, 310(3):630-641, 2010. 1, 1.1

[27] R. Montgomery. Hamiltonicity in random graphs is born resilient. Journal of Combinatorial Theory, Series B, 139:316-341, 2019. 1

[28] R. Montgomery. Spanning trees in random graphs. Advances in Mathematics, 356:106793, 2019. 1.1, 6

[29] R. Montgomery. Hamiltonicity in random directed graphs is born resilient. Combinatorics, Probability and Computing, 29(6):900-942, 2020. 1

[30] R. Nenadov and N. Škorić. Powers of Hamilton cycles in random graphs and tight Hamilton cycles in random hypergraphs. Random Structures \& Algorithms, 54(1):187-208, 2019. 1.1

[31] R. Nenadov and N. Škorić. On Komlós' tiling theorem in random graphs. Combinatorics, Probability and Computing, 29(1):113-127, 2020. 1.1, 8

[32] R. Nenadov, A. Steger, and M. Trujić. Resilience of perfect matchings and Hamiltonicity in random graph processes. Random Structures \& Algorithms, 54(4):797-819, 2019. 1

[33] V. Rödl, A. Ruciński, and E. Szemerédi. A Dirac-type theorem for 3-uniform hypergraphs. Combinatorics, Probability and Computing, 15(1-2):229-251, 2006. 1.1

[34] A. Scott. Szemerédi's regularity lemma for matrices and sparse graphs. Combinatorics, Probability and Computing, 20(3):455-466, 2011. 2.5

[35] P. Seymour. Problem section. In Combinatorics: Proceedings of the British Combinatorial Conference, pages 201-202, 1973. 1

[36] N. Škorić, A. Steger, and M. Trujić. Local resilience of an almost spanning $k$-cycle in random graphs. Random Structures \&5 Algorithms, 53(4):728-751, 2018. 1

[37] B. Sudakov. Robustness of graph properties. In Surveys in combinatorics 2017, volume 440 of London Math. Soc. Lecture Note Ser., pages 372-408. Cambridge Univ. Press, Cambridge, 2017. 1

[38] B. Sudakov and V. H. Vu. Local resilience of graphs. Random Structures Ef Algorithms, 33(4):409-433, 2008. 1,1

[39] A. Thomason. Pseudo-random graphs. In Random graphs '85 (Poznań, 1985), volume 144 of North-Holland Math. Stud., pages 307-331. North-Holland, Amsterdam, 1987. 1

[40] A. Thomason. Random graphs, strongly regular graphs and pseudorandom graphs. Surveys in Combinatorics, 123:173-195, 1987. 1

Institute of Mathematical Sciences, Tel Aviv University, Tel Aviv, Israel

Email address: moussetf@inf.ethz.ch

Institute of Theoretical Computer Science, ETH Zürich, 8092 Zürich, Switzerland

Email address: nskoric@inf.ethz.ch

Institute of Theoretical Computer Science, ETH Zürich, 8092 Zürich, Switzerland

Email address: mtrujic@inf.ethz.ch 